\title{
1 Predictive simulation of non-steady-state transport of gases through rubbery polymer membranes
}

3 Marielle Soniat, ${ }^{\mathrm{a}, \mathrm{b}}$ Meron Tesfaye ${ }^{\mathrm{c}, \mathrm{d}}$, Daniel Brooks, ${ }^{\mathrm{e}}$ Boris Merinov, ${ }^{\mathrm{e}}$ William A. Goddard, III, ${ }^{\mathrm{e}}$

4 Adam Weber $^{\mathrm{a}, \mathrm{c}}$ and Frances A. Houle*a,b

5 a Joint Center for Artificial Photosynthesis, Lawrence Berkeley National Laboratory, Berkeley, 6 CA 94720

$7 \quad{ }^{\mathrm{b}}$ Chemical Sciences Division, Lawrence Berkeley National Laboratory, Berkeley, CA 94720

$8{ }^{\mathrm{c}}$ Energy Storage and Distributed Resources Division, Lawrence Berkeley National Laboratory, 9 Berkeley, CA 94720

$10{ }^{\mathrm{d}}$ Department of Chemistry and Chemical Engineering, University of California, Berkeley, CA 1194720

$12{ }^{\mathrm{e}}$ Materials and Process Simulation Center (MSC), Beckman Institute, California Institute of 13 Technology, Pasadena, CA, 91125

14 * Author to whom correspondence should be addressed. fahoule@lbl.gov, (510) 495-8135.

\section{Abstract:}

16 We develop a multiscale, physically-based reaction-diffusion kinetics model for non-steady-state

17 transport of simple gases through a rubbery polymer. The rubbery polymer case applies to

18 membrane applications where high permeability is desired or has developed due exposure to plasticizers such as $\mathrm{CO}_{2}$. To construct a model that has no adjustable parameters, we utilize experimental data from the literature as well as new measurements of non-steady-state permeation. We have also performed molecular dynamics simulations of collisions of $\mathrm{CO}_{2}$ with the surface of PDMS to obtain an estimate of the gas-polymer sticking probability. The model successfully reproduces time-dependent experimental data for two distinct systems: (1) $\mathrm{O}_{2}$ quenching of a phosphorescent dye embedded in poly(n-butyl(amino) thionylphosphazene), and (2) $\mathrm{O}_{2}, \mathrm{~N}_{2}, \mathrm{CH}_{4}$ and $\mathrm{CO}_{2}$ transport through poly(dimethyl siloxane). The modeling study provides new insights to microscopic aspects of permeant transport through rubbery polymers and is used to predict selectivity targets for two gas separation applications. 
Table of Contents Figure:

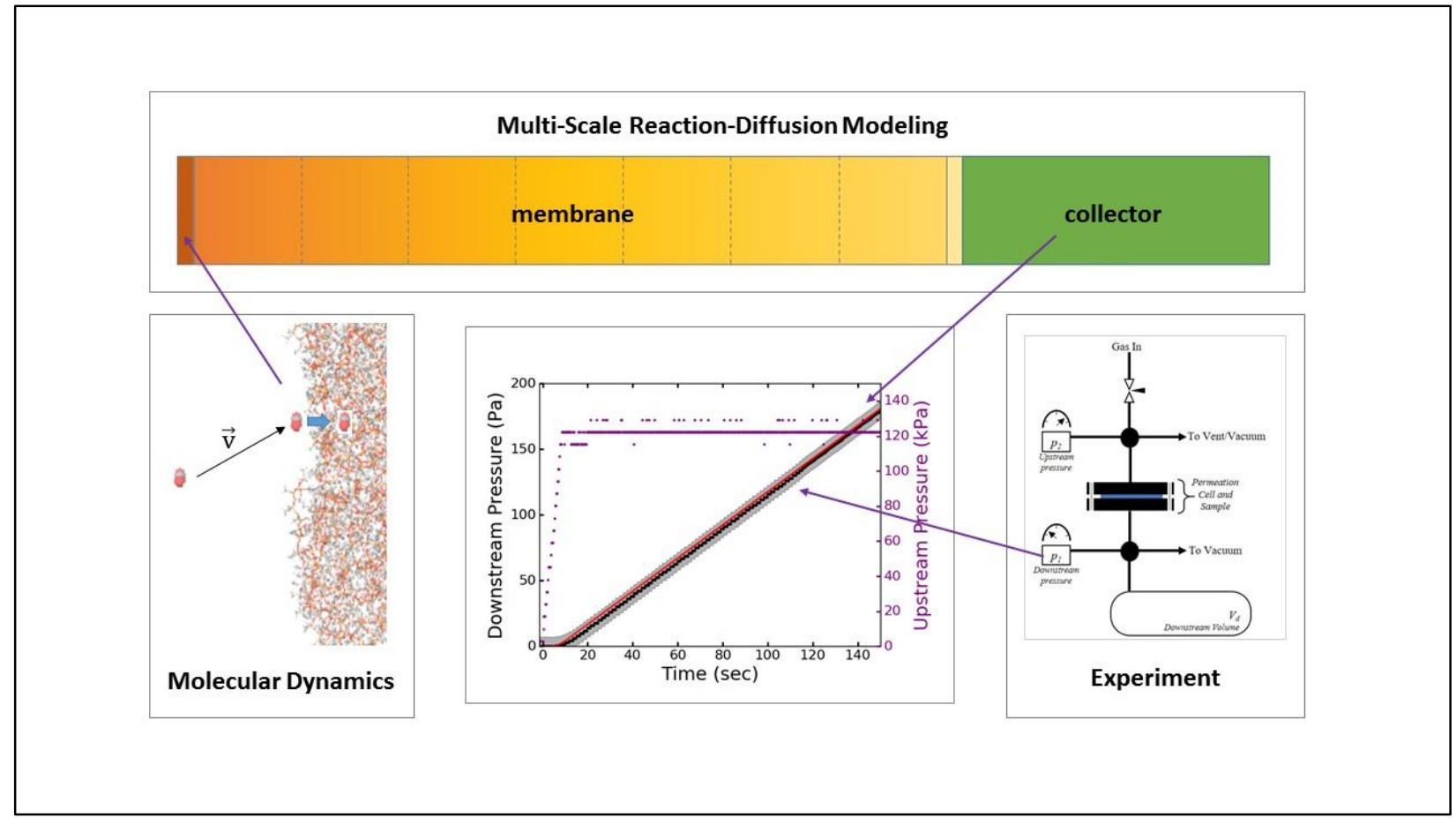

31 


\section{Introduction}

Permeant transport through a polymer membrane is driven by differences in pressure, concentration, temperature, and, if it is a polyelectrolyte, the electrical potential across it. ${ }^{1}$ When the concentration of a permeant is constant on the upstream and downstream sides of the membrane, steady-state transport is described by the solution-diffusion model, and is defined in terms of the permeability, $P_{m}$. The permeability is the product of the Henry's Law solubility, $S$, and diffusivity, $D$, i.e. $P_{m}=S \times D .{ }^{2}$ Polymeric membrane materials fall into two basic classes, highly permeable rubbers with low glass transition temperatures $(\mathrm{Tg})$ and weakly permeable glasses with high Tg. The solution-diffusion model applies to both. The physical processes that take place in the polymer bulk do not depend on whether the permeant was initially present as a component of a liquid or a gas phase. The details of the uptake process, on the other hand, are likely to depend on phase. The solution-diffusion model assumes that no matter what the source of the permeant, its uptake at the polymer surface is much faster than diffusion through its bulk, ${ }^{3}$ and therefore is not rate limiting. Molecular dynamics studies and kinetics models using parameterized schemes have provided important insights to the detailed nature of uptake and permeation through the membrane bulk via tortuous paths, for ion-conducting, phase-separated perfluorosulfonic acid polymers. ${ }^{4-5}$ This level of physical detail, however, is not available for most membrane systems.

Steady-state permeation is relevant to many continuous applications using polymeric membranes such as purification and electrolyzer systems, but not to those in which permeant concentrations and other operating conditions such as temperature vary significantly with time. Examples are gas sensor and intermittent gas separations systems, electrolyzers using electricity from renewable sources, or photoelectrochemical systems that use sunlight to convert components of air and water into fuels. The membrane composition and structure required for each of these applications varies and the physical details governing their time-dependent performance cannot be assumed to be simply captured as they are in the solution-diffusion model. Accordingly, in this study we focus on one extreme - inert gases permeating a rubbery polymer - to begin to develop a model description of behaviors far from steady state. The basic model is constructed in a manner that enables it to be extended to more complex situations such as mixed-matrix membranes, metal form separators, permeant-induced ageing, permeation of mixtures whose transport properties through the membrane are not independent of each other, and permeation under variable temperature conditions. The ability to predictively model such situations will allow membrane architectures well-suited for time-varying applications to be 66 developed.

The rubbery, non-interacting regime applies to gas sensors and certain gas separators. We consider time-dependent permeation through two types of rubbery systems: (1) reversible permeation of gases into a gas-sensor system, and (2) pre-steady-state transport through a gas separator. Time-dependent data for a sensor system have been reported in the literature. ${ }^{6-8}$ For the present work, we examine a comprehensive data set describing quenching of phosphorescence of a dye in a series of rubbery polymers by $\mathrm{O}_{2}$ and have used them to develop and validate a basic model framework. Time-dependent gas separator data obtained under wellcontrolled conditions are scarce, so we have measured non-steady-state permeation of gases over a range of pressures through a rubbery polymer, polydimethylsiloxane (PDMS) for this work. The gases - $\mathrm{O}_{2}, \mathrm{~N}_{2}, \mathrm{CO}_{2}$, and $\mathrm{CH}_{4}$ - are selected to span a range of gas solubilities and nature of 
interaction with the polymer chains. We have examined aspects of uptake by using molecular

78 dynamics simulations to estimate the fraction of $\mathrm{CO}_{2}$-PDMS collisions that result in adsorption

79 or absorption. A multiscale, reaction-diffusion model framework has been constructed that successfully reproduces both sensor and separator data with no adjustable parameters, and reveals several aspects of the permeation process that are kinetically significant. Because it is predictive, the model is useful to examine functional systems. We describe simulation results for use of rubbery membranes to separate gas mixtures, and product crossover between cathode and anode chambers during a diurnal cycle of a solar-driven photoelectrochemical $\mathrm{CO}_{2}$ reduction system. The latter case is applicable to a system in which the membrane has become rubbery due to prolonged exposure to $\mathrm{CO}_{2}$.

\section{II. Methods and Materials}

Three separate methodologies have been combined in the present study: stochastic reactiondiffusion simulations, molecular dynamics simulations, and gas permeation measurements. The materials set considered in this work is shown in Figure 1.

\section{A. Reaction-diffusion simulations}

The goal of this work is to develop a predictive model whose simulation outputs can be compared directly to experimental data. This requires that all simulation inputs (system geometry, rate constants and concentrations of all reactants and products) be measured

97

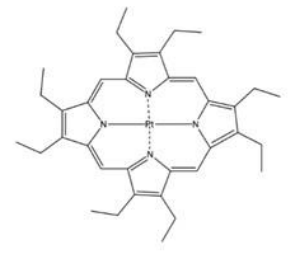

(a) platinum octaethyl porphyrin

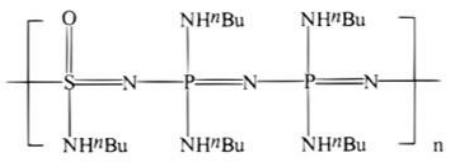

(c) Poly[n-butyl(amino)thionylphosphazene] 
Figure 1. Chemical structures of the phosphorescent dye (a) platinum octa-ethyl porphyrin (PtOEP), and the polymers (b) poly(dimethyl siloxane) (PDMS) and (c) poly[nbutyl(amino)thionylphosphazene] (C4PTP) considered in this work.

experimentally, calculated by molecular dynamics simulations, or derived from established theory. The simulation reaction-diffusion code used in this study incorporates stochastic methods, a type of kinetic Monte Carlo that produces an accurate timebase. ${ }^{9-10}$ It provides a rigorously accurate solution to the master equation for Markov systems, and is a useful alternative to coupled differential equation integrators for complex materials systems. It is

108 particularly well-suited for systems that require detail at both nanoscale and macroscale dimensions, and involve large dynamic ranges in rates. The open access code package is Kinetiscope. ${ }^{11}$ Details on its computational methodology and applications to various types of condensed organic phase reaction-diffusion systems are described in previous publications. ${ }^{12-15}$ The basic framework used in this work is a 1-dimensional reaction-diffusion system comprising a stack of micro-scale compartments to represent the polymer bulk, and source and receiver compartments separated by 1-nm-thick interfacial regions from the membrane. The construction of the reaction-diffusion model is described in Section III.

\section{B. Molecular Dynamics Simulations}

Typically, gas uptake and desorption processes at the surface of a polymer membrane are considered to be instantaneous, with bulk transport being the controlling factor in permeation rate. However, to build a predictive model, it is necessary to use physically based rate constants for all processes. Gas-rubbery polymer collision dynamics are not well studied, so we have selected one of the gases used in this work, $\mathrm{CO}_{2}$, for investigation of the uptake process using molecular dynamics (MD) simulations and applied the results to other gases studied in this work.

The initial PDMS structure is generated using standard procedures. First, a low-density ( $\rho$ $\left.=0.0245 \mathrm{~kg} / \mathrm{m}^{3}\right)$, structure is created with 25 chains of PDMS of 100-monomer length. The system contains 25,053 atoms in total. To ensure that there are no overlapping atoms in the structure, 100 energy minimization steps and $10 \mathrm{ps}$ of dynamics in the NVT ensemble ${ }^{16}$ at $10 \mathrm{~K}$ are performed. The density of the structure is increased by running $500 \mathrm{ps}$ of dynamics in the NPT ensemble using the algorithm of Martyna, Tobias, and Klein (MTK). ${ }^{17}$ To ensure entanglement of the polymers, $2000 \mathrm{ps}$ of dynamics is performed in the NVT ensemble with a Nosé-Hoover thermostat and with long range interactions reduced to $20 \%$ of their initial value, in accordance with the Scaled Effective Solvent (SES) procedure. ${ }^{18}$ Finally, to prevent high-energy structures, energy minimization is performed for $300 \mathrm{ps}$ and the lattice parameters of the structure are again relaxed with $200 \mathrm{ps}$ of NPT dynamics.

This procedure results in a roughly $70-\AA$ thick slab of PDMS created with dimensions of $6.79 \times 6.79 \times 6.79 \mathrm{~nm}^{3}$. To create a PDMS surface, the length of the cell is increased by $200 \AA$ in the $\mathrm{x}$-direction to generate a region of empty space. All polymer chains are kept intact. The surface is then equilibrated for $3000 \mathrm{ps}$ in the NVT ensemble using the Berendsen thermostat at $300 \mathrm{~K}$. This procedure results in a bulk density of $\approx 0.985 \mathrm{~kg} / \mathrm{m}^{3}$, which is above the experimental reference value of $0.970 \mathrm{~kg} / \mathrm{m}^{3},{ }^{19}$ but below the experimental sample densities of 1.06 to 1.08

$141 \approx 0.94 \mathrm{~kg} / \mathrm{m}^{3}$ because of the surface roughness. The instantaneous surface is designated using the 
method of Willard and Chandler, ${ }^{20}$ which is shown in Figure 2 and described more fully in the SI.

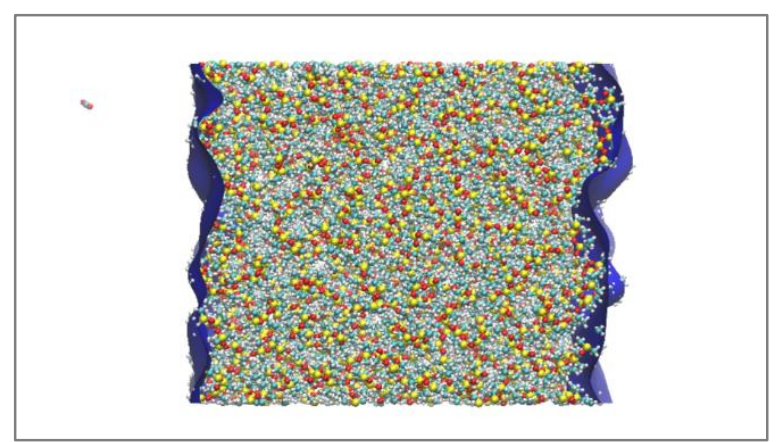

Figure 2. The structure of poly(dimethyl siloxane) (PDMS) in the molecular dynamics simulations. Hydrogen atoms are shown in white, carbon in light blue, oxygen in red and silicon in yellow. The instantaneous interface is shown in blue. The $\mathrm{CO}_{2}$ molecule is sent towards the surface of the PDMS polymer structure for an adsorption simulation.

\begin{abstract}
$\mathrm{CO}_{2}$ absorption, adsorption, and desorption events are tracked using a procedure based on the work of Julin et al. ${ }^{21-22} \mathrm{~A} \mathrm{CO}_{2}$ molecule is introduced at a distance of approximately $15 \AA$ from the surface and assigned a velocity from the Maxwell-Boltzmann distribution at $300 \mathrm{~K}$, with the constraint that the $\mathrm{x}$-component of the velocity vector is within a 45-degree cone pointed toward the surface. After 100 ps of simulation time, the outcome (adsorption, absorption, desorption) is recorded based on the position of the $\mathrm{CO}_{2}$ molecule relative to the surface, defined as points within $4 \AA$, i.e. twice the van der Waals radius, of the instantaneous surface. Several other distances are used as the adsorption cutoff to test the sensitivity of the sticking coefficient to this parameter. The adsorption simulations are performed in the NVE ensemble.
\end{abstract}

Simulations are performed using the Desmond MD simulation package and the OPLS2005 force field. A time-step of $1 \mathrm{fs}$ is used for short-range interactions and a 3 fs time-step is used for long-ranged interactions. Long-ranged electrostatics are computed using the Ewald summation. A short-ranged Coulomb cutoff of $9 \AA$ is used. Center of mass motion is removed at each time step in the adsorption simulations.

\title{
C. Experimental permeation measurements
}

Complete time-dependent permeation curves for several gases through PDMS were obtained in this work as described in this section. To our knowledge such data have not been previously reported, but they are needed for accurate model construction and validation. Additional details are presented in the SI.

Sample Preparation. PDMS samples are prepared using standard procedures. The PDMS base and a proprietary crosslinker (Sylgard 184, Dow Corning Corp., Auburn, MI) are mixed in a 10:1 ratio by weight. The PDMS solution is degassed to remove air bubbles and cast onto silicon wafers to the desired thickness via the spin-coating method. The polymer films are stored in deionized water until use. Prior to measurement, each sample is thoroughly dried and degassed. 
The thickness of the dry films is in the range of 163 to $165 \mu \mathrm{m}$, as measured by a micrometer (Mitutoyo, Aurora, IL). A helium gas displacement pycnometer (AccuPyc II 1340 from Micromeritics Instrument Corp., Norcross, GA) is used to measure the density, which ranges from 1.06 to $1.08 \mathrm{~kg} / \mathrm{m}^{3}$. The glass transition temperature is $149 \pm 0.3 \mathrm{~K}$, measured using differential scanning calorimetry (DSC800 Perkin Elmer, Waltham, MA). experiments. ${ }^{23} \mathrm{~A}$ schematic is available in Reference MT-REF. Prior to the permeation experiment, gas is evacuated from the entire apparatus, a check for leaks is performed, and the measured permeabilities are corrected for this leak. Upstream pressures of 119 to $835 \mathrm{kPa}$ are tested. The upstream pressure is maintained at least two orders of magnitude greater than the downstream pressure during the measurements so that the pressure difference across membrane is effectively constant. The upstream pressure is monitored using a Honeywell STJE (Honeywell, Morris Plains, NJ) pressure transducer, which has a range of 200 to $7000 \mathrm{kPa}$ and an accuracy of $\pm 100 \mathrm{kPa}$. The downstream pressure is monitored using a Baratron MKS (MKS Instruments, San Jose, CA) pressure transducer, which has a range of 100 to $1000 \mathrm{~Pa}$ and an accuracy of $\pm 7 \mathrm{~Pa}^{24}$ The temperature of the system is maintained at $308 \mathrm{~K}$ in a water bath using an Alpha immersion thermostat (Lauda, Delran, NJ). All gases, $\mathrm{N}_{2}, \mathrm{O}_{2}, \mathrm{CH}_{4}$, and $\mathrm{CO}_{2}$, (Praxair, Danbury, CT) are at least $99.9 \%$ pure and are used as received.

After the gas permeation process has reached steady state, the gas permeability of the 196 membrane, $P_{m}$, is calculated from the gas flux, $J$, through membrane of thickness, $l$, due to a difference in partial pressure, $\Delta p$, across the membrane:

$$
P_{m}=\frac{J l}{p_{u s}-p_{d s}}=\frac{V_{d s} l}{\Delta p A R T}\left[\left(\frac{d p_{d s}}{d t}\right)_{s s}-\left(\frac{d p_{d s}}{d t}\right)_{\text {leak }}\right]
$$

where $V_{d s}$ is the downstream collection volume, $A$ is the membrane area, $R$ is the universal gas constant, $T$ is the temperature, $p_{d s}$ refers to the downstream pressure, $p_{u s}$ refers to the upstream pressure, and $\left(d p_{d s} d d t\right)_{S S}$ is the steady-state pressure rise, which is corrected for the leak rate $\left(d p_{d s} d d\right)_{\text {leak }}$. At steady state, $P_{m}=D x S$.

\section{Development of a kinetic model for permeation of a rubbery polymer}

Detailed reaction-diffusion models for non-steady state transport in polymer membranes have not been reported previously, and development of a predictive description requires multiple independent types of time-dependent experimental measurements to validate it. ${ }^{12,14}$ There are few of these in the literature for any polymer type, however a particularly useful set for rubbery polymers has been reported by Jayarajah and coworkers. ${ }^{6-7}$ In this section we describe how the model for their measurements, quenching of a phosphorescent dye in a rubbery polymer by oxygen for a sensor application, ${ }^{8}$ has been developed and compare predictions to experimental observations. The polymer used is poly(n-butyl (amino) thionyl phosphazene) (C4PTP) This polymer has a low Tg of $257 \mathrm{~K}$ and was studied at a thickness of $163 \mu \mathrm{m}$. The dye platinum octaethyl porphyrin (PtOEP) is embedded within the polymer, and quenching of its laser-excited phosphorescence acts as an indicator of the internal concentration of $\mathrm{O}_{2}$ gas. The structures of the C4PTP monomer and of PtOEP are shown in Figure 1.

\section{A. System Geometry}

As shown in Figure 3, the membrane is represented as a set of 100 homogeneous compartments, each $1.63 \mu \mathrm{m}$ thick, coupled by diffusion paths. An interfacial compartment of 1 
$\mathrm{nm}$ thickness is provided at the gas-polymer interface to enable explicit description of adsorption, desorption and transfer of gases to and from the polymer bulk. The other side of the membrane is in contact with a glass substrate and is not permeable. The area of the membrane is taken to be the footprint of the laser beam used for dye excitation, $2 \mathrm{~mm}$ by $1 \mathrm{~mm}$.

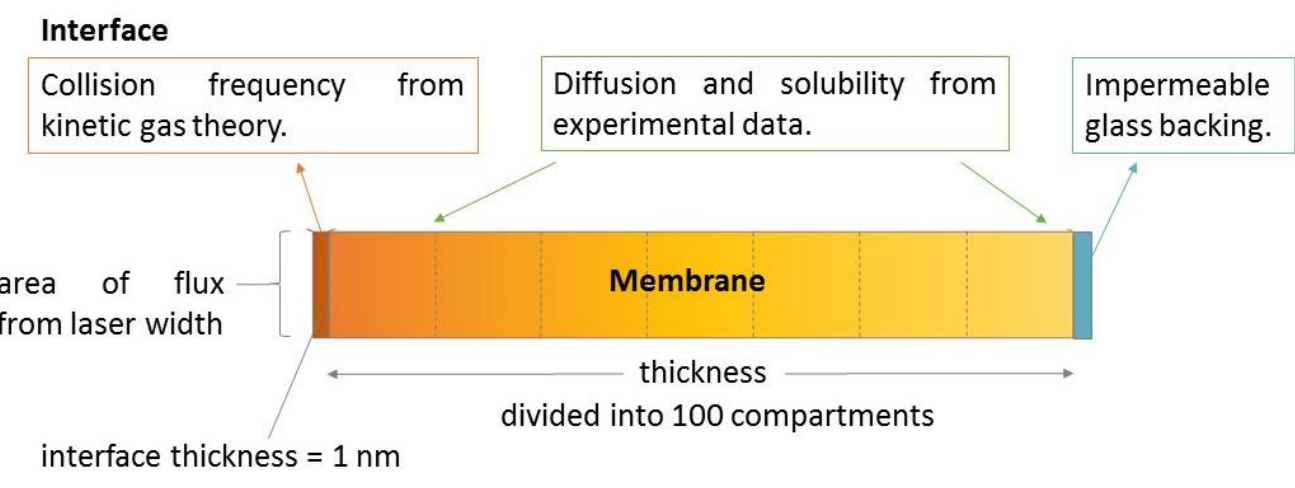

Figure 3. Overview of multi-scale model for a sorption simulation. The membrane is divided into 100 compartments, plus one thin interfacial compartment.

\section{B. Gas-polymer interactions}

The experimental diffusion coefficient is used for gas transport throughout the polymer, including the surface layers. The maximum concentration of a gas in the membrane is determined by its experimental Henry's Law solubility.

Adsorption and desorption are represented as direct processes involving a gas molecule and a surface site, a description used in other studies. ${ }^{15}$ Absorption is broken into two steps: sticking of the gas to the membrane surface and diffusion from the surface layer into the bulk. Sticking is represented as a reaction between a gas molecule and an empty surface site,

$$
\text { gas }+ \text { site } \stackrel{k_{a d s}}{\longrightarrow} \text { gas - surface }
$$
where the adsorption rate constant, $k_{a d s}$, is the collision frequency of the gas with the surface, $k_{\text {coll }}$, at the appropriate partial pressure, times the sticking probability, $\mu$,

$$
k_{\text {ads }}=\mu k_{\text {coll }}=\mu(Z A)=\mu\left(\frac{p}{\sqrt{2 \pi m_{w} k_{B} T}}\right) A
$$

where $Z$ is the collision frequency per unit area, $A$ is the experimental surface area, $p$ is the upstream partial pressure of the gas, $m$ is the molecular mass of the gas, $k_{B}$ is Boltzmann's constant, and $T$ is the temperature. ${ }^{25}$ The sticking coefficient is initially assumed to be 0.1 ; the sensitivity of the simulation to this value has been investigated and will be discussed later in this section. We use the generic term "site" because we assume that the gas molecules can physisorb to an atom type at the surface. The concentration of surface sites is assumed to be that of surface atoms in a liquid, $10^{14}$ atoms $/ \mathrm{cm}^{2}{ }^{26}$ Since the surface is represented as a volume, the sites are distributed through a $1 \mathrm{~nm}$ thickness, giving a concentration of $1.66 \mathrm{moles} /$ Liter. Once adsorbed, 
the gas molecule can be absorbed by diffusing from the interface layer to the adjacent bulk polymer compartment. Desorption from the membrane is represented as detachment from a surface site. Because the polymer and gas molecules are weakly interacting, we assume that there are only van der Waals bonds between the gas and the polymer; thus desorption rate constant, $k_{d e s}=1.4 \times 10^{11} \mathrm{~s}^{-1}$, is based on the breaking of a single van der Waals bond in the gas phase. ${ }^{27-28}$ The sensitivity of the simulation results to all these assumptions is discussed in the SI.

\section{Kinetics of dye quenching}

The dye is assumed to be uniformly distributed throughout the polymer bulk and its excitation/deexcitation reactions are:

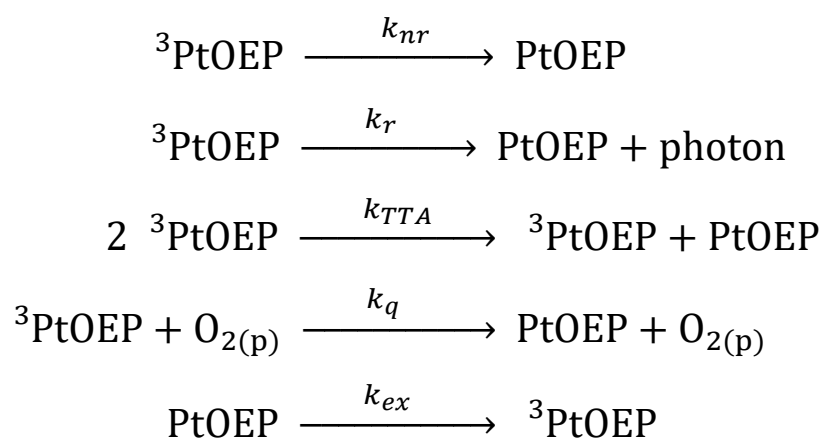

where PtOEP is in the ground state, ${ }^{3} \mathrm{PtOEP}$ is in the first excited triplet state, and $\mathrm{O}_{2(\mathrm{p})}$ is the oxygen gas which has been absorbed into the polymer. These steps are the non-radiative decay, radiative decay, triplet-triplet annihilation, quenching, and excitation steps, respectively. In this section we describe how the rate constants have been determined for these steps.

The photophysics were not fully described in the experimental studies, ${ }^{7}$ so they have been determined using other work on PtOEP in polymer environments. ${ }^{29-30}$ From the ground state, PtOEP absorbs at $380 \mathrm{~nm}$ and $547 \mathrm{~nm}$ to enter the first excited state ${ }^{30}$ greater than $99.9994 \%$ of PtOEP molecules excited to the first singlet state undergo intersystem crossing to the first excited triplet state ${ }^{3} \mathrm{PtOEP} .{ }^{29}$ This allows fluorescence from the singlet state to be neglected. PtOEP emits at $647 \mathrm{~nm}$ from the triplet state when well-dispersed in solution or embedded in a clear polymer ${ }^{29-30}$ For excited-state dye at high concentration or in solution with low viscosity, triplettriplet annihilation may occur. Triplet-triplet annihilation (TTA) is the process in which one triplet state PtOEP molecules quenches another and is itself excited back to the first excited singlet state. Because each excited singlet quickly relaxes to the first excited triplet we do not explicitly track the first excited singlet state in Reaction (V). ${ }^{29}$

Two types of experiments are examined to construct and validate a kinetic model for PtOEP photoprocesses. One determines the dye's phosphorescence lifetime using pulsed laser excitation, ${ }^{29-30}$ the other tracks the intensity of phosphorescence from continuous laser excitation of PtOEP as a function of time, $I(t)$, during absorption and desorption of gases. ${ }^{7}$

\section{Dye lifetime by pulsed laser excitation.}

Phosphorescence decay. Simulations of phosphorescence decay in the absence of $\mathrm{O}_{2}$ were performed first in order to establish the dye kinetics. The reported values for the quantum yield and rate constants for Reactions II-V are listed in Table 1. For these simulations, we can omit 
Reaction VI and start with a population of excited dye molecules. As shown in Figure 4, and explained in the SI, simulations using these values and the reaction scheme (steps II-V) are in good agreement with experiment only if the concentration of initially excited state dye molecules, $\left[{ }^{3} \mathrm{PtOEP}\right]_{\text {init }}$, is lower than expected based on the amount of dye added to the polymer. $\left[{ }^{3} \mathrm{PtOEP}\right]_{\text {init }}$ is calculated using

$$
\left[{ }^{3} \text { PtOEP }\right]_{\text {init }}=\frac{[\text { PtOEP }] \sigma_{a} \omega_{L}}{h v_{L}}
$$

282

283

284

where [PtOEP] is the total concentration of dye molecules, $\sigma_{a}$ is the absorption cross section of the dye at the exciting wavelength, $\omega_{L}$ is the energy density of the laser beam, $h$ is Planck's constant, and $v_{L}$ is the exciting frequency. For the experiments of Bansal et al. $\left(\sigma_{a}=4.70 \times 10^{-21}\right.$ $\left.\mathrm{m}^{2} ; \omega_{L}=17.0 \mathrm{~J} / \mathrm{m}^{2} ; v_{L}=8.64 \times 10^{14} \mathrm{~s}^{-1} ;[\mathrm{PtOEP}]=0.0731 \mathrm{M}\right)$, this calculation predicts that $3 \%$ of the total number of dye molecules will be excited during the laser pulse. However, simulations revealed that this value leads to a decay rate that is too fast. Simulations and experiment could be brought into agreement using a value of $0.8 \%$ of the total dye for $\left[{ }^{3} \mathrm{PtOEP}\right]_{\text {init. }}$. Data for lifetimes in the presence of air are also reported. The introduction of oxygen in air opens a channel for quenching which competes with phosphorescence. Simulations using the reported quenching rate constant (see Table 1) show that in this case a value of $0.8 \%$ also applies. The reason for this difference is not clear, but may reflect dye aggregation since clusters of PtOEP emit at $790 \mathrm{~nm},{ }^{29-}$ ${ }^{30}$ which would not be detected in the experiments. Sensitivity studies for $\left[{ }^{3} \mathrm{PtOEP}\right]$ init are described in the SI.

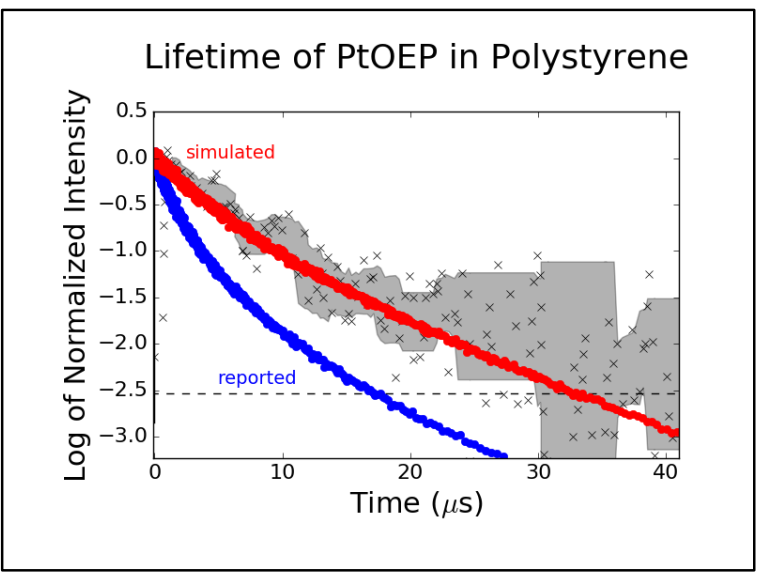

Figure 4. Simulated and experimental phosphorescence decay in air. The experimental data points from Ref. ${ }^{29}$ are marked with black x's, and the gray region indicates the local standard deviation (over 11 data points). The dashed line indicates the lower detection limit of the instrument. The blue markers are for the simulation data using the reported value of $\left[{ }^{3} \mathrm{PtOEP}\right]_{\text {init }}$ $=9.96 \times 10^{-3} \mathrm{M}$. The red markers are for a simulation using a reduced value of $\left.\left[{ }^{3} \mathrm{PtOEP}\right]\right]_{\text {init }}=$ $5.85 \times 10^{-4} \mathrm{M}$.

Quenching. Quenching in a polymer is expected to be diffusion controlled and so should be different for C4PTP in which $\mathrm{O}_{2}$ has a higher diffusivity than in PS. The quenching rate constants for PtOEP by $\mathrm{O}_{2}$ were not reported in Jayarajah's study although pulsed laser 
excitation was used to measure a lifetime for PtOEP in both a de-oxygenated sample and at external partial pressure of oxygen $p_{O 2}=21 \mathrm{kPa}$, the same partial pressure at which the sorption experiments are done. ${ }^{7}$ Combining the lifetime measured for de-oxygenated C4PTP, $\tau_{0}$, with the quantum yield in PS, $\phi_{\mathrm{P}}$ (assumed to be the same for C4PTP), allows us to calculate $k_{r}$ as

$$
k_{r}=\frac{\phi_{P}}{\tau_{0}}
$$

311 and $k_{n r}$ as

$$
k_{n r}=\frac{1-\phi_{P}}{\tau_{0}}
$$

312 The quenching rate constant, $k_{q}$, is calculated from the lifetime measured at $p_{O 2}=21 \mathrm{kPa}$, by 313 assuming that TTA is negligible. Then,

$$
k_{q}=\frac{\frac{1}{\tau}-k_{r}-k_{n r}}{\left[O_{2(p)}\right]}
$$

314

where $\left[\mathrm{O}_{2(\mathrm{p})}\right]$ is the concentration of oxygen gas within the polymer. The value for $k_{q}$ calculated as a diffusion-controlled reaction using the Smoluchowski equation is of the same order of magnitude. However, this equation contains a parameter that could not be completely constrained by theory, so this method was not used. A summary of the rate constants and other physical characteristics used in the simulation of the dye-polymer system are presented in Table 1 .

Table 1. Simulation inputs for phosphorescence quenching. Values for polystyrene are from Ref. ${ }^{29}$ unless otherwise noted. The values for C4PTP are from Ref. ${ }^{7}$ unless otherwise noted. The radiative and non-radiative decay rate constants for C4PTP are calculated with an assumed $\phi_{\mathrm{P}}=$ 0.54 (see text for details). [ $\left.{ }^{3} \mathrm{PtOEP}\right]_{\mathrm{init}}$ are my estimates for the pulsed laser experiments, based on simulations ( $10 \%$ of reported $\left[{ }^{3} \mathrm{PtOEP}\right]_{\text {init }}$ in Ref. $\left.{ }^{29}\right)$. These simulations are carried out in a single well-mixed compartment.

\begin{tabular}{|l|l|l|l|}
\hline & & $\mathrm{PS}$ & $\mathrm{C} 4 \mathrm{PTP}$ \\
\hline $\mathrm{D}\left(\mathrm{O}_{2}\right)$ & $\mathrm{m}^{2} / \mathrm{s}$ & $1 \times 10^{-11}(\mathrm{a})$ & $4.0 \times 10^{-10}$ \\
\hline $\mathrm{S}\left(\mathrm{O}_{2}\right)$ & $\mathrm{M} / \mathrm{Pa}$ & $8.26 \times 10^{-8}(\mathrm{a})$ & $1.18 \times 10^{-8}$ \\
\hline$\left[\mathrm{O}_{2}\right]_{\max }$ & $\mathrm{M}$ & $1.73 \times 10^{-3}$ & $2.40 \times 10^{-4}$ \\
\hline$[\mathrm{PtOEP}]$ & $\mathrm{M}$ & 0.0731 & $3.1 \times 10^{-4}$ \\
\hline$\left.{ }^{3} \mathrm{PtOEP}\right]_{\text {init }}$ & $\mathrm{M}$ & $5.85 \times 10^{-4}$ & $2.48 \times 10^{-6}$ \\
\hline$\phi \mathrm{P}$ & & 0.54 & 0.54 \\
\hline$\tau_{0}$ & $\mu \mathrm{s}$ & 60 & 103 \\
\hline$k_{r}$ & $\mathrm{~s}^{-1}$ & $8.33 \times 10^{3}$ & $3.9 \times 10^{3}$ \\
\hline$k_{n r}$ & $\mathrm{~s}^{-1}$ & $8.30 \times 10^{3}$ & $5.8 \times 10^{3}$ \\
\hline
\end{tabular}




\begin{tabular}{|l|l|l|l|}
\hline$k_{\text {TTA }}$ & $\mathrm{M}^{-1} \mathrm{~s}^{-1}$ & $4 \times 10^{7}$ & $4 \times 10^{7}$ \\
\hline$k_{q}$ & $\mathrm{M}^{-1} \mathrm{~s}^{-1}$ & $1.82 \times 10^{7} *$ & $3 \times 10^{9}$ \\
\hline
\end{tabular}

$328 *$ calculated from the reported pseudo-first order rate constant $3.20 \times 10^{4} \mathrm{~s}^{-1}$ and the expected concentration of $\mathrm{O}_{2}$ in the polymer.

\section{Continuous excitation-deexcitation of the dye}

Excitation rate constant. Time-dependent $\mathrm{O}_{2}$ sorption measurements for PtOEP in C4PTP are made using continuous illumination of the sample exposed to a fixed external oxygen pressure $p_{O 2}=21 \mathrm{kPa}^{6-7}$ The time-dependent intensity detected, $I(t)$, is reported relative to the ratio $B+1=I_{0} / I_{e q}$ where $I_{0}$ is the phosphorescence intensity in the absence, of oxygen, and $I_{e q}$ is the intensity for a constant concentration of $\mathrm{O} 2$ consistent with $p_{\mathrm{O} 2}=21 \mathrm{kPa}$. The relevant dye kinetics are established by the lifetime measurements in the previous section except for the dye excitation rate constant $k_{e x}$ (Reaction VI). The absorbed power density of the continuous wave laser used in the experiments was not reported by Jayarajah et al. ${ }^{6-7}$ Since $k_{r}, k_{n r}, k_{q}$, and $k_{T T}$ in the dye kinetics scheme are constrained, we can use simulations of $I_{0}$ and $I_{e q}$ to estimate $k_{e x}$. Steady-state values for $B+1$ are plotted as a function of assumed $k_{e x}$ in Figure S3. The phosphorescence intensity ratio is quite insensitive to $k_{e x}$ over a range of 1 and $100 \mathrm{~s}^{-1}$, so the median of $k_{e x}=50 \mathrm{~s}^{-1}$ is used. In this range of $k_{e x}$, the simulated $B+1=66$ close to the experimental range of $B+1=75 \pm 6$. The difference may be due to experimental parameters leading to a slightly higher concentration of $\mathrm{O}_{2}$ being present within the polymer than expected, or a lower photon detection efficiency.

Time-dependent data. The intensity over time, $I(t)$, was used by Jayarajah et al. to determine the solubility coefficient, $S$, and the diffusion coefficient, $D$, for $\mathrm{O}_{2}$ in C4PTP using a two-parameter fit to the equation,

$$
I(t)=\frac{I_{0}}{l} \int_{0}^{l} \frac{1}{1+B \xi(x, t)} d x
$$

350 where

$$
B=\frac{I_{0}}{I_{e q}}-1=4 \pi N_{A v} \tau_{0} R_{A B}\left(D_{O_{2}} S_{O_{2}}\right) p_{O_{2}}
$$

351 and

$$
\xi(x, t)=\frac{Q(x, t)}{Q_{e q}}
$$

where $l$ is the membrane thickness, $N_{A v}$ is Avogadro's number, and $\tau_{0}$ is the lifetime of PtOEP in the polymer environment in the absence of oxygen. $\xi(x, t)$ is the relative concentration of $\mathrm{O}_{2}$ within the membrane at position $x$ at time $t$, whereas $Q(x, t)$ is the absolute concentration of $\mathrm{O}_{2}$ within the membrane at position $x$ at time $t$, and $Q_{e q}$ is the equilibrium concentration of $\mathrm{O}_{2}$ within the membrane. ${ }^{6-7,32}$ The equation above assumes that the dye is both distributed and excited homogeneously throughout the membrane (i.e. the sample is optically thin and Beer's law does 
not apply). In the simulations, we also use a homogeneous dye distribution and assume homogeneous excitation.

The continuous excitation experiments were performed in two modes: (i) sudden

\section{Simulations of $\mathrm{O}_{2}$ sorption and phosphorescent dye quenching in a sensor system}

Simulations of emitted light intensity as $\mathrm{O}_{2}$ sorbs into or desorbs from a polymer are used to calculate $I(t)$ for comparison to experimental data. The absorption and desorption simulation results are compared with the experimental data in two ways: (i) the total amount of $\mathrm{O}_{2}$ predicted to be absorbed in the polymer in the simulation is used to calculate the expected $I(t)$ using the reported intensity ratio, ${ }^{7} B+1$, and equations $7-9$, and (ii) full simulation of the dye kinetics and the photons produced per unit time are directly compared to the experimental intensity over time. The use of two methods serves as a check on the internal consistency of the experimental data and on the validity of the reaction mechanism.

The simulations predict the amount of oxygen gas within the membrane at all times. We compare the simulated $\mathrm{O}_{2}$ amount to experiment, which does not measure $\mathrm{O}_{2}$ in the film directly, by calculating $N$, the expected amount of $\mathrm{O}_{2}$ in the membrane, from the experimental $I(t)$ curve. We assume $d x=l$ in Equation 7, i.e. that the film is entirely uniform. The maximum amount of $\mathrm{O}_{2}$ in the membrane, $N_{\max }$, is found at equilibrium. $N_{\max }=Q_{e q} * V$, where $V=d y * d z * l$ and the equilibrium concentration of $\mathrm{O}_{2}$ is $Q_{e q}=S * p_{O 2}$. Then, rearranging Eq 7-9 gives

$$
N=N_{\max } \frac{1}{B}\left[\frac{B+1}{I(t)}-1\right]
$$

Simulation results for sorption and desorption performed using the reaction scheme developed in the previous section are compared to experiment in in Figure 5. The shape of the calculated curves is in good agreement with observations, but there are several quantitative differences. The initial rise in the simulated amount of $\mathrm{O}_{2}$ in the polymer shown in Figure $5 \mathrm{~b}$ is faster than that calculated from the measured $I(t)$ using Equation 10. In the simulation, the amount of $\mathrm{O}_{2}$ in the membrane compartments near the gas-polymer interface increases rapidly, and they become saturated with $\mathrm{O}_{2}$. The rate of $\mathrm{O}_{2}$ absorption then slows, limited by $\mathrm{O}_{2}$ diffusion to the other side of the membrane. The reason for the difference between simulated and experimental amount of $\mathrm{O}_{2}$ is unclear. One possibility is that the assumption of $d x=l$ in the 
395 calculation of the amount of $\mathrm{O}_{2}$ from $I(t)$ is not valid, which is supported by the fact that 396 simulated and measured $I(t)$ curves are in much closer agreement (Figure 5a).

397

398

(a)

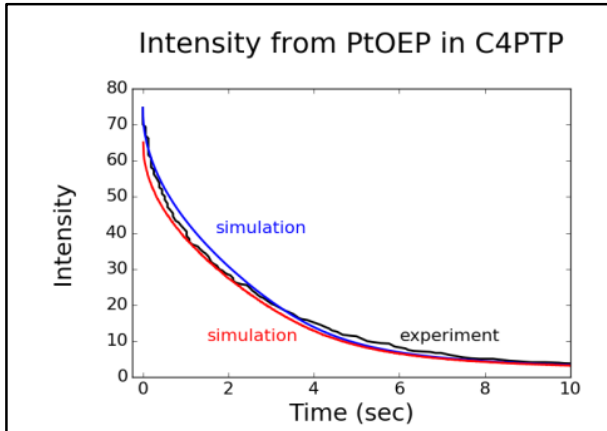

Jayarajah_Fig4-6aDye_perm.rxn (Row 1 Layer 1) - Concentration of Species 02-bsite

399

(c)

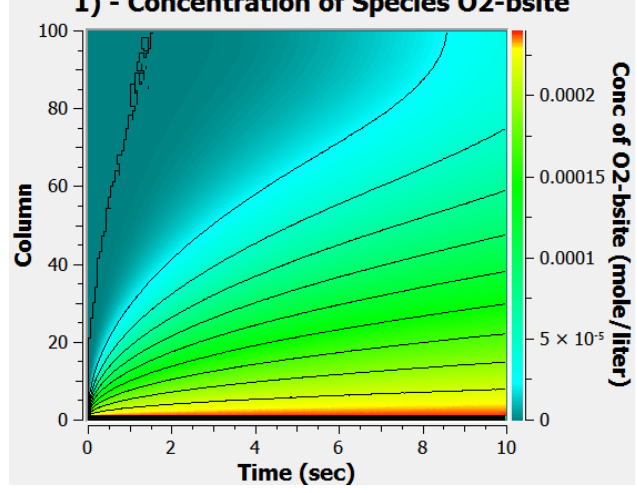

(d)

(b)

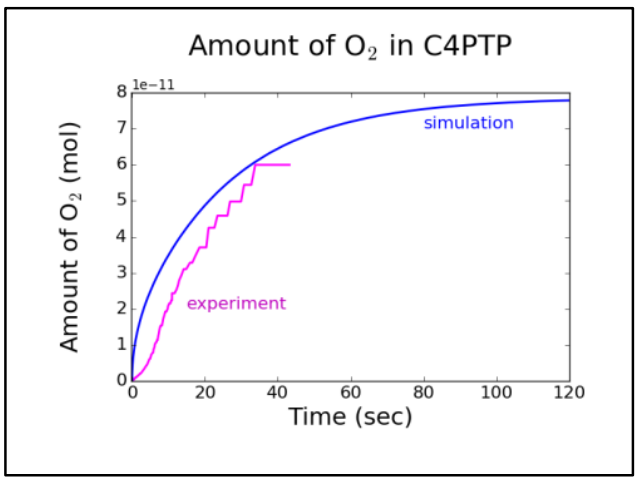

Jayarajah_Fig4-6aDye_perm.rxn (Row 1 Layer 1) - Concentration of Species PtOEP*
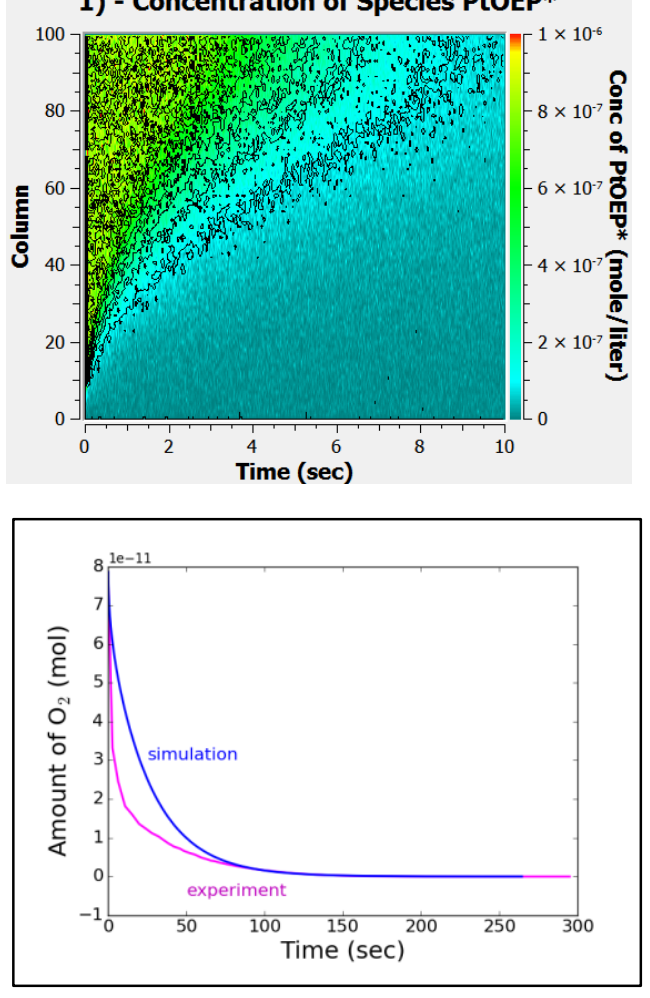

400

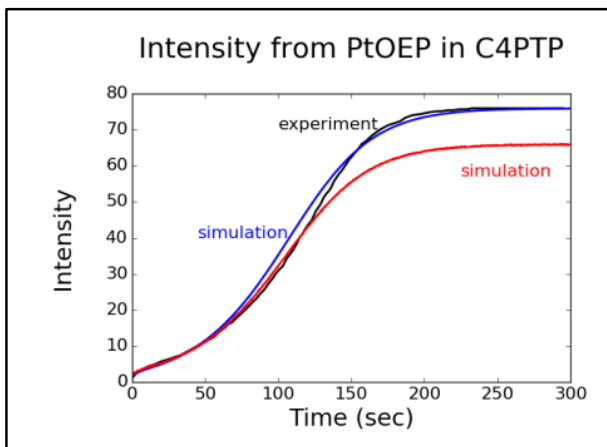

(e)

(f) 

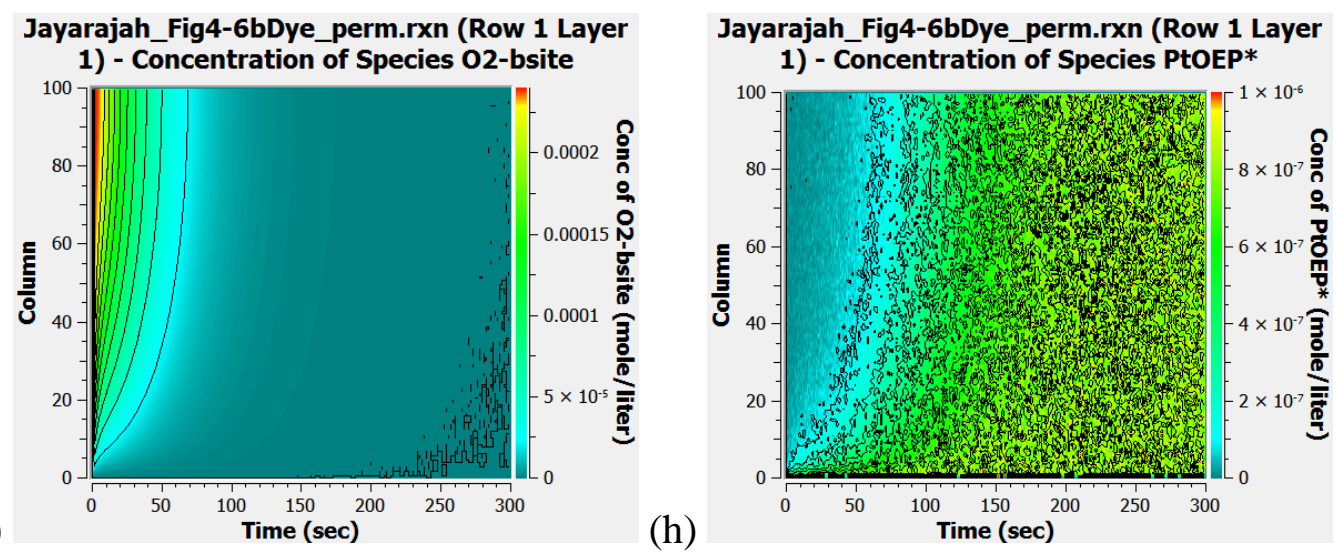

402 Figure 5. Results of simulation of C4PTP. (a) $I(t)$ is calculated from the oxygen concentration with Eqn. 7 - 9 (blue) and from simulation of the dye photophysics (red) as $\mathrm{O}_{2}$ sorbs into the polymer. (b) The amount of $\mathrm{O}_{2(\mathrm{p})}$ in the polymer increases during sorption. (c) The concentration of $\mathrm{O}_{2(\mathrm{p})}$ through the polymer thickness over time during sorption. (d) The concentration of ${ }^{3} \mathrm{PtOEP}$ through the polymer thickness over time during sorption. (e) $I(t)$ is calculated from the oxygen concentration with Eqn. 7 - 9 (blue) and from simulation of the dye photophysics (red) as $\mathrm{O}_{2}$ desorbs from the polymer. (f) The amount of $\mathrm{O}_{2(\mathrm{p})}$ in the polymer decreases during desorption. (g) The concentration of $\mathrm{O}_{2(\mathrm{p})}$ through the polymer thickness over time during desorption. (h) The concentration of ${ }^{3} \mathrm{PtOEP}$ through the polymer thickness over time during desorption.

Another possibility is the presence of inhomogeneities either in the polymer, in the dye distribution, or in emission, that would lead to the same total phosphorescence intensity but no simple relationship between light emission and total $\mathrm{O}_{2}$ in the film. This possibility was tested in two ways. First, a simulation in which dye is not present in the top quarter of the membrane gives a similar $I(t)$ curve to experiment, showing that emission intensity is insensitive to spatial distribution of the dye and therefore to details of the $\mathrm{O}_{2}$ kinetics. Second, altering the pressure profile of $\mathrm{O}_{2}$ so that it does not instantaneously rise to $p_{O 2}=21 \mathrm{kPa}$, but rather increases over several seconds, which is more similar to the experimental procedure, does not change the shape of the curve. These considerations suggest that Equations 7-9 are qualitatively valid for estimating the full time dependence of $\mathrm{O}_{2}$ absorption, but may not be quantitative in all cases. Comparison of Figure 5a and Figure 5b also shows that while phosporescence decays rapidly, the $\mathrm{O}_{2}$ concentration in the membrane does not reach saturation until much later. In the sorption simulations, $\mathrm{O}_{2}$ starts to reach the membrane compartments furthest from the interface after 2.0 sec. The concentration of ${ }^{3} \mathrm{PtOEP}$ is very small, and is fully quenched $8.1 \mathrm{sec}$ after the introduction of $\mathrm{O}_{2}$ to the polymer. However, the concentration of $\mathrm{O}_{2}$ continues to rise for another $140 \mathrm{sec}$. Because the concentration of excited state dye is so low, there is not a one-to-one correspondence between the intensity and the $\mathrm{O}_{2}$ concentration in the sensor film outside the range of 1 to $50 \mu \mathrm{M}$ of $\mathrm{O}_{2}$. The distribution of $\mathrm{O}_{2}$ through the membrane over time is shown in Figure $5 \mathrm{c}$. This can be contrasted with the concentration of ${ }^{3} \mathrm{PtOEP}$ through the membrane over time is shown in Figure $5 \mathrm{~d}$.

The shape of the $I(t)$ desorption curve for C4PTP is also captured well by the simulation (Figure 5e). There is an initial small but rapid increase in $I(t)$ because the $\mathrm{O}_{2}$ in the top 
membrane compartments desorb in the first few seconds. From then until $50 \mathrm{sec}$, there is slower rise in intensity as $\mathrm{O}_{2}$ diffuses from the lower compartments and desorbs, but the overall $\mathrm{O}_{2}$ concentration within the membrane is still high enough to quench most of the dye. A more rapid increase in intensity ensues after $50 \mathrm{sec}$, once the $\mathrm{O}_{2}$ concentration is no longer in excess of that needed to quench ${ }^{3} \mathrm{PtOEP}$. Finally, the maximum intensity is reached around $200 \mathrm{sec}$, once all the $\mathrm{O}_{2}$ is removed from the polymer. Note that this description is observed in the simulation, shown in Figures $5 \mathrm{~g}$ and $5 \mathrm{~h}$, not just inferred. The predicted $\mathrm{O}_{2}$ content shown in Figure $5 \mathrm{f}$ is higher than that extracted from the $I(t)$ measurements. This is likely to have the same origin as discussed above for the sorption case, Figure $5 b$.

Sensitivities of the simulation results to uncertainties have been thoroughly evaluated. Those for $D$ and $S$ are discussed in the SI and shown in Figure S4. Those of the assumed properties of the gas-polymer -interface are presented in Figures S5 and S6 and summarized here. Saturation of surface sites by $\mathrm{O}_{2}$, assuming a sticking probability of 0.1 , occurs within $10^{-21}$ seconds at $21 \mathrm{kPa}$. Reduction of the sticking coefficient to $10^{-17}$ has no effect on the $\mathrm{O}_{2}$ profile, nor does increasing the sticking coefficient to 1 . The concentration of adsorption or desorption sites can also be varied by 1 to 2 orders of magnitude with no effect. Alteration of the surface diffusion coefficient does not change the sorption results. Reduction, though not increase, of the surface diffusion coefficient does alter the desorption. Finally, the desorption rate constant can also be reduced or increased by several orders of magnitude without altering the oxygen profiles. Because the simulation results are largely insensitive to surface kinetics at the rubbery polymerinert gas interface, this type of simulation is insufficient to learn about them.

\section{Permeation of gases through a membrane separator, experiment and simulations}

Having established a suitable reaction-diffusion framework as described in the preceding sections, we use it to examine the fully time-dependent permeation data obtained in this work, capturing not only steady state permeation, but also downstream pressure rise for steady-state and pre-steady-state regimes. In this section, we present the permeation measurements, and simulations of them. In view of the insensitivity of sorption of gas by rubbery polymer sorption to assumptions of gas sticking, we report molecular dynamics calculations to provide a description of this process for one system, thus avoiding an arbitrary guess in the model.

\section{A. Measurements of time-dependent permeation through PDMS}

In this study, the permeation of the gases $\mathrm{N}_{2}, \mathrm{O}_{2}, \mathrm{CO}_{2}$, and $\mathrm{CH}_{4}$ through PDMS is investigated at $308 \mathrm{~K}$ for various pressure gradients. The pressure $v s$ time data are presented in the SI. The permeability is calculated from the rate of pressure increase downstream from the membrane when steady-state is reached, and differences due to the experimental setup are accounted for in the calculation of permeation via Equation 2. The permeability values as a function of pressure gradient are given in Figure 6. Each data point represents an average value of three or more different samples and measurements. The leak rate for each measurement is kept under $0.05 \%$ of the permeate flux. Propagated measurement error ranges from $1-4 \%$. The error bars show the sample-to-sample variability, which is $10-20 \%$. As can be seen in Figure 6, permeability shows no dependence on pressure gradient, indicating that the sorption isotherm is also linear. Permeation values for PDMS can vary with change in base to cross-linker ratio, casting method, and annealing temperature. Our measured values are consistent within experimental error and variability of literature values. ${ }^{33-35}$ Higher pressure gradients $(600-850$ 
$\mathrm{kPa}$ ) were also investigated, but the variation was not as well characterized, and so this data is omitted from Figure 6.

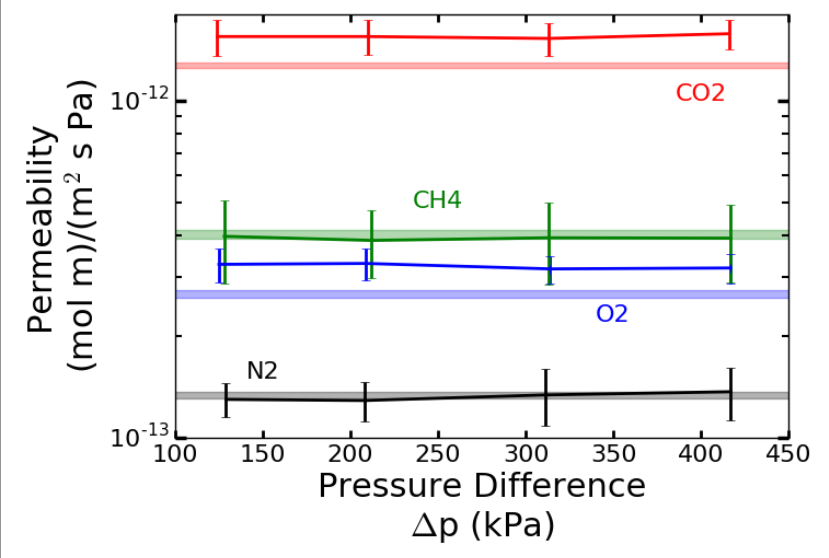

483 Figure 6. Comparison of experimental permeability to literature values. The literature values 484 from Ref. ${ }^{33}$ are shown with their standard deviation as the shaded regions.

\section{B. Calculation of sticking probability of $\mathrm{CO}_{2}$ on PDMS}

Because the sticking of a gas molecule to the rubbery polymer surface was found to be not kinetically limiting, molecular dynamics is used to investigate the adsorption and sticking process for one gas, $\mathrm{CO}_{2}$, and assumed to be applicable to the other gases investigated. The results from 250 simulations of $\mathrm{CO}_{2}$ impacts onto a PDMS surface are shown in Figure 7 and summarized in Table II. Some care must be taken in the interpretation of these results: the distinction between an adsorbed and absorbed molecule is arbitrary, especially for atoms just below the interface, and the fate of molecules adsorbed on the surface is not clear from the finite simulation time. Thus, the fraction of absorbed molecules, which is $30 \%$, can be taken as a lower bound of the sticking coefficient, and the fraction of absorbed plus adsorbed molecules, which is $50 \%$, can be taken as an upper bound. Since few data are available on gases sticking to PDMS or other rubbers, we use the MD data for $\mathrm{CO}_{2}$ as a first reasonable guess at the sticking coefficient for any weakly interacting gas onto a rubber. The minimum sticking coefficient of $30 \%$ is used in the stochastic simulations for all gas molecules. 
(a)

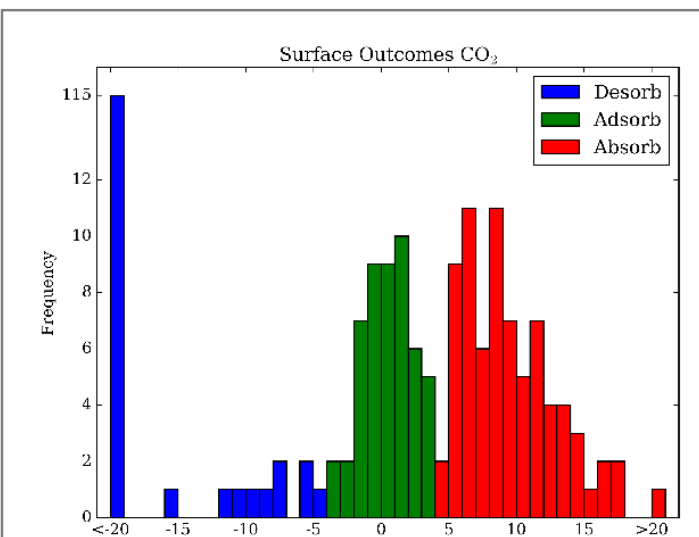

Position Relative to Surface $(A)$

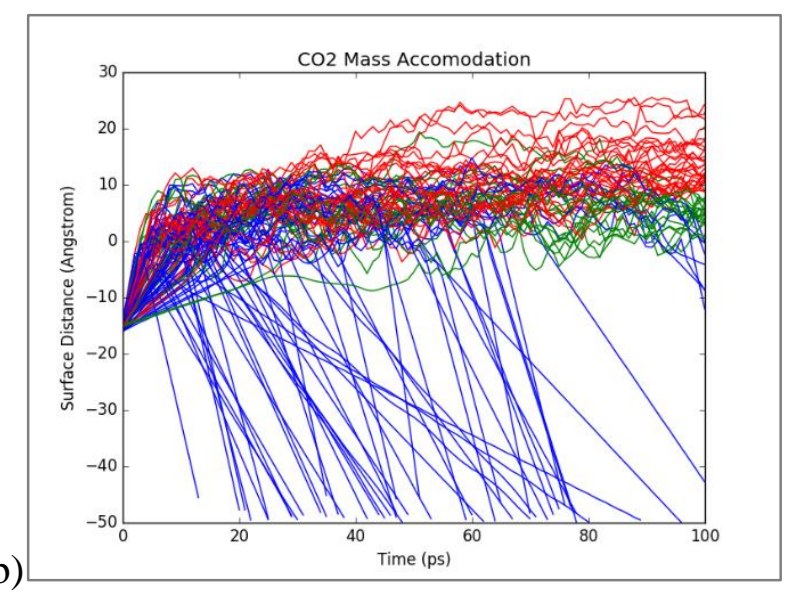

(b)

502 Figure 7. Results of molecular dynamics simulations for CO2 sticking to PDMS. Absorbed molecules are plotted in red, adsorbed molecules in green, and scattered and desorbed molecules in blue. (a) Histogram showing the distribution of outcomes from all 250 simulations. Note that the far left blue bar represents desorption in 115 simulations. (b) Distance from the surface as a function of time (for 100 randomly selected trajectories).

Table 2. Results of Molecular Dynamics study of sticking of $\mathrm{CO}_{2}$ to a PDMS surface.

\begin{tabular}{|c|c|c|c|c|}
\hline Events & Absorb & Adsorb & Desorb & Total \\
\hline Number & 75 & 50 & 125 & 250 \\
\hline Percent & 30 & 20 & 50 & 100 \\
\hline
\end{tabular}

\section{Simulations of time-dependent permeation}

The model framework constructed for phosphorescence quenching (Figure 3) is modified for PDMS permeation by removing the dye quenching kinetics and replacing the glass support with a gas collector volume. A $1 \mathrm{~nm}$ thick interfacial region is provided between the polymer and the gas collector, and the desorption steps are moved from the upstream interface to the downstream one. The size of the collection compartment is set equal to the experimental collection volume of $41.3 \mathrm{~cm}^{3}$. The multi-scale simulation setup is shown in Figure 8. Instrumental characteristics such as volumes and the measured pressure rise in the upstream chamber when gas is initially introduced are included in the model. 


\begin{tabular}{l} 
Interface \\
\begin{tabular}{|l|} 
Collision frequency from \\
kinetic gas theory.
\end{tabular} \\
\hline
\end{tabular}

kicking coefficient from

Sticking coefficient from molecular dynamics.

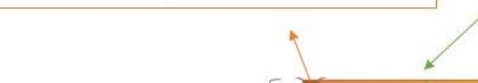

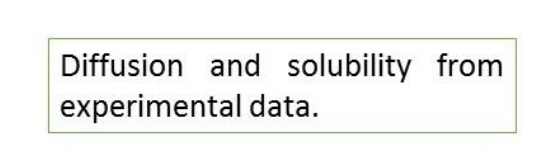

Interface

Desorption rate constant from Arrhenius equation.

The solubility of each gas is assumed to be the literature value. ${ }^{33}$ The permeability measured in each experiment is divided by the literature solubility to calculate the diffusivity. We use the literature value for solubility because solubility varies only over an order of magnitude for a given polymer, whereas the diffusivity within the polymer may vary over six orders of magnitude for different permeants. ${ }^{36}$ Values for $\mathrm{S}$ and $\mathrm{D}$ used in this work are presented in Table 3.

Figure 8. Overview of multi-scale model for a permeation simulation. The membrane is divided into 100 compartments, plus two thin interfacial compartments. An additional compartment collects gas after its permeation.

Table 3. Input values for multi-scale simulations for comparison to experiment. The following are constant across all simulation of experimental permeation data: the surface site concentration is $1.66 \mathrm{M} ; \mathrm{V}_{\mathrm{ds}}=4.13 \times 10^{-5} \mathrm{~m}^{3} ; k_{\text {des }}=1.4 \times 10^{11} \mathrm{~s}^{-1}$; diffusion of gases in the gas phase of 2.0 $\mathrm{m}^{2} / \mathrm{s}$. The thickness is divided into 100 compartments. The adsorption rate constant is calculated from Eqn. 2 with $\mu=0.30$. Sample L4S2 has a thickness of $163 \mu \mathrm{m}$ and an area of $0.203 \mathrm{~cm}^{2}$. Sample L5S1 has a thickness of $165 \mu \mathrm{m}$ and an area of $0.187 \mathrm{~cm}^{2}$.

\begin{tabular}{|l|l|l|l|l|l|l|l|}
\hline gas & $\mathrm{S}$ & $\begin{array}{l}\text { sample } \\
\text { ID }\end{array}$ & pus & $\mathrm{D}$ & $k_{\text {act }}$ & $k_{\text {leak }}$ & $\begin{array}{l}\text { initial } \\
{[\mathrm{gas}]_{\mathrm{ds}}}\end{array}$ \\
\hline & $\mathrm{M} / \mathrm{Pa}$ & & $\mathrm{kPa}$ & $\mathrm{m}^{2} / \mathrm{s}$ & $\mathrm{M}^{-1} \mathrm{~s}^{-1}$ & $\mathrm{M}^{-1} \mathrm{~s}^{-1}$ & $\mathrm{M}$ \\
\hline $\mathrm{CO}_{2}$ & $5.68 \times 10^{-7}$ & $\mathrm{~L} 4 \mathrm{~S} 2$ & 122 & $2.34 \times 10^{-9}$ & 0.02 & $5.44 \times 10^{-11}$ & $1.04 \times 10^{-7}$ \\
\hline & & & 206 & $2.34 \times 10^{-9}$ & 0.017 & $5.17 \times 10^{-11}$ & $1.04 \times 10^{-7}$ \\
\hline & & & 311 & $2.39 \times 10^{-9}$ & 0.015 & $4.72 \times 10^{-11}$ & $1.04 \times 10^{-7}$ \\
\hline $\mathrm{N}_{2}$ & $3.96 \times 10^{-8}$ & $\mathrm{~L} 4 \mathrm{~S} 2$ & 122 & $3.27 \times 10^{-9}$ & 0.0015 & $1 \times 10^{-12}$ & $2.08 \times 10^{-7}$ \\
\hline & & & 206 & $3.26 \times 10^{-9}$ & 0.0016 & $1 \times 10^{-12}$ & $1.56 \times 10^{-7}$ \\
\hline & & & 416 & $3.26 \times 10^{-9}$ & 0.0024 & $1 \times 10^{-12}$ & $1.56 \times 10^{-7}$ \\
\hline
\end{tabular}




\begin{tabular}{|l|l|l|l|l|l|l|l|}
\hline & & L5S1 & 820 & $2.80 \times 10^{-9}$ & 0.0004 & $1 \times 10^{-12}$ & 0 \\
\hline
\end{tabular}

Experimentally, the upstream permeant gas pressure increases over several seconds after opening the gas valve, and is tracked. The pressure vs time curve is used as an input to the simulation, and the beginning of its rise is used as the zero for the simulation time base. The internal concentration of gas in a polymer is proportional to the external pressure, $p_{u s}$,

$$
\left[\operatorname{gas}_{(p)}\right]=S p_{u s}
$$

where the proportionality constant, $S$, is the gas's Henry's law solubility and is constant over the pressure range in this study. ${ }^{33} \mathrm{We}$ treat this by increasing the maximum possible concentration of the gas in the membrane in concert with the increase in external pressure, assuming instantaneous equilibration between the two. Such a dynamic treatment is necessary for agreement between simulation and experiment before steady state is reached, especially for the more permeable gases or where the upstream pressure rise takes place over a long time. More details on implementation of this part of the model are presented in the SI.

Figures 9 and 10 show the permeation curves from experiment and the corresponding simulations for $\mathrm{N}_{2}$ and $\mathrm{CO}_{2}$, respectively, through PDMS for upstream pressures from 100 to $800 \mathrm{kPa}$. The figures for these gases are highlighted here because they represent the extremes in permeability among the gases tested; additional data on $\mathrm{O}_{2}$ and $\mathrm{CH}_{4}$ is available in the SI. The agreement at all times, gas types, and pressures is within experimental error, confirming that the simulation framework and the model represent the full permeation process over a wide range of conditions, Henry's law solubilities, and permeabilities.

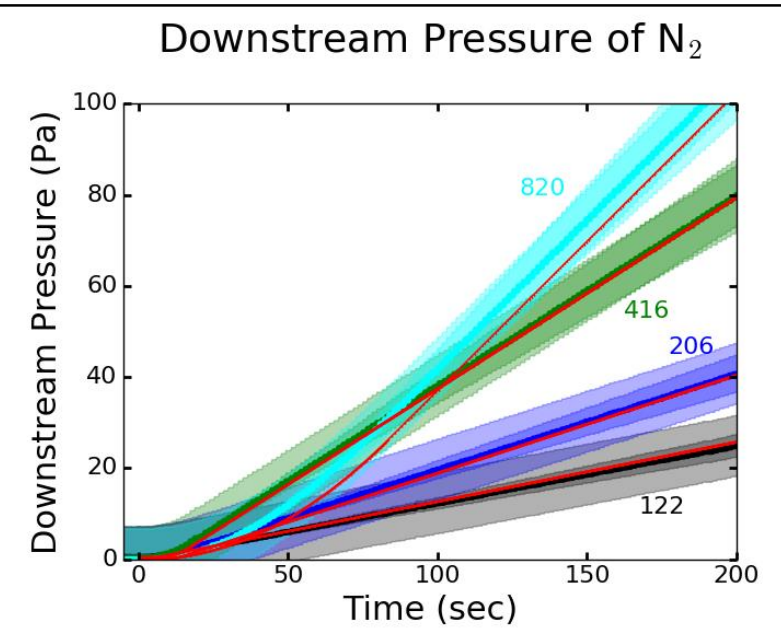

(a) 

(b)

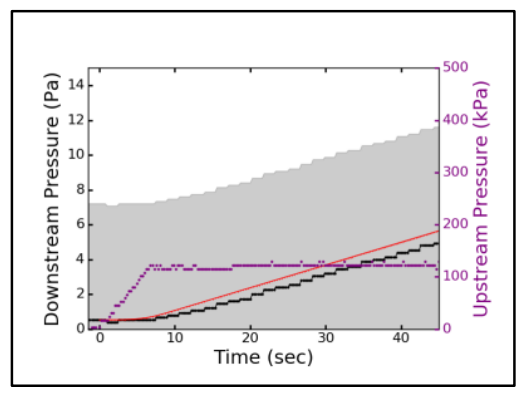

(c)

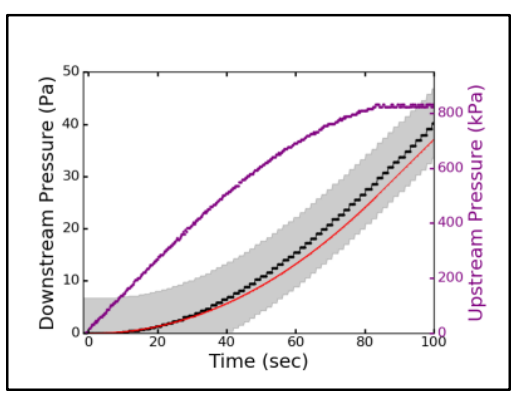

560 Figure 9. Comparison of simulation and experiment permeation curves for $\mathrm{N}_{2}$. The simulation results are shown in red. (a) All pressures. The shaded regions represent an experimental sampleto-sample standard deviation of $10 \%$. The curves are labeled with the upstream pressure in $\mathrm{kPa}$. (b) Pre-steady state for $\mathrm{p}_{\mathrm{us}}=122 \mathrm{kPa}$ (c) Pre-steady state for $\mathrm{p}_{\mathrm{us}}=820 \mathrm{kPa}$. The shaded region represents the accuracy of the downstream pressure transducer. The lower limit of detection of the downstream pressure detector is $0.13 \mathrm{~Pa}$, resulting in jumps in the downstream pressure in

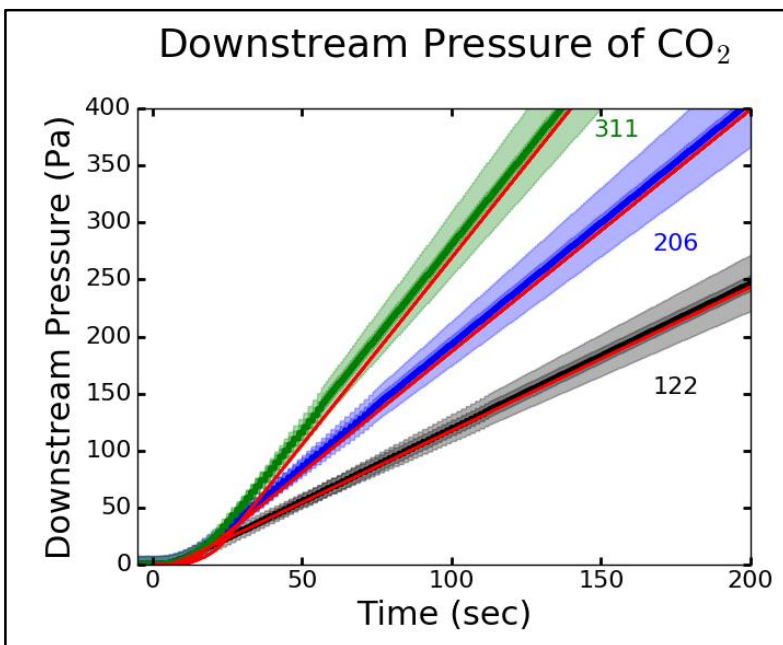
the figures showing pre-steady state. The experimental upstream pressure rise is shown in purple.

569

570

571

572

573

574

575

576 (a)

(b)

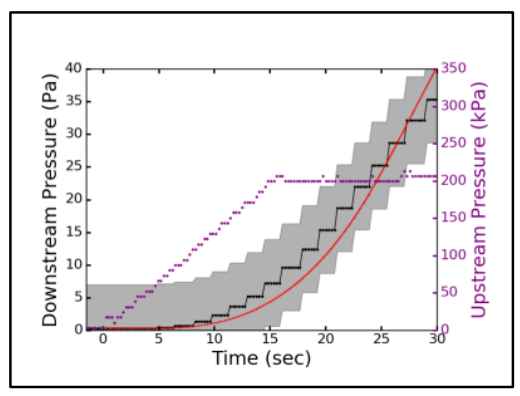

Figure 10. Comparison of simulation and experiment permeation curves for $\mathrm{N}_{2}$. The simulation results are shown in red. (a) All pressures. The shaded regions represent an experimental sampleto-sample standard deviation of $10 \%$. The curves are labeled with the upstream pressure in $\mathrm{kPa}$. (b) Pre-steady state for $\mathrm{p}_{\mathrm{us}}=206 \mathrm{kPa}$. The shaded region represents the accuracy of the downstream pressure transducer. The lower limit of detection of the downstream pressure detector is $0.13 \mathrm{~Pa}$, resulting in jumps in the downstream pressure in the figures showing presteady state. The experimental upstream pressure rise is shown in purple. 


\section{Predictive simulations for two rubbery membrane separations systems}

The reaction-diffusion framework developed in this work is used to develop two models that the entire permeation process, both steady-state and pre-steady-state for experimental data sets involving rubbery polymers: phosphorescence quenching, and permeation through a membrane. The models have no adjustable parameters and are physically realistic, and therefore predictive. Having been validated, we use the framework for PDMS to examine two other types of systems at an exploratory level: $\mathrm{CO}_{2}$ separations from air, and solar $\mathrm{CO}_{2}$ photoelectrochemical reduction systems. In this section we discuss the relevant physical properties of the membrane system captured by the model, then describe the simulation results.

\section{A. Core physical characteristics of the PDMS permeation model}

Both experimental and simulation results for permeation of simple gases through PDMS presented in this work are consistent with previous studies, and add two new insights to dynamical aspects of the system. First, we find that inclusion of instantaneous increases in the maximum possible concentration as the permeant pressure increases on the upstream side is required for good agreement between the experimental and calculated pressure rise for all gases studied. While the computational treatment for single permeants is straightforward in this case, it is likely to be more complex when time-varying mixtures are involved. Second, we find that the bulk PDMS diffusion coefficient is sufficient to describe gas motion at all locations within the polymer. This indicates that tortuosity, polymer chain fluctuations, and other detailed, molecularlevel characteristics are well-captured by the Fickian diffusion description, and do not need to be included explicitly.

Because the permeation process in the rubbery system was found to be insensitive to gaspolymer interfacial kinetics, MD simulations are used to obtain information about this process for one case, $\mathrm{CO}_{2}$ absorption into PDMS, and show that the gas sticking probability is in the range of $30 \%$ to $50 \%$. The most similar system which has been studied experimentally is the scattering of the $\mathrm{O}_{2}$ gas from the surface of the hydrocarbons squalane and dodecane. ${ }^{37}$ At incident energies of $8 \mathrm{~kJ} / \mathrm{mol}$, twice the average kinetic energy for gas molecules in this study, the oxygen molecules fully transfer their excess energy to the hydrocarbon surface, ${ }^{37}$ indicating a sticking probability near 100\%. A MD study of Ar colliding with hydrocarbon self-assembled monolayers ${ }^{38}$ show that the hydrocarbon chains are able to redistribute the energy of the incoming molecule on the same timescale as the impact of the atom with the surface by recruiting a large number of low-frequency vibrational modes. ${ }^{38}$ PDMS similarly contains a large number of low-frequency interactions, and so a similar energy transfer mechanism may apply. Thus, we conclude that a sticking probability of $30 \%$ to $50 \%$ is reasonable for a light, inert gas molecule at ambient temperature colliding with a flexible polymer surface, though further study on this type of system would be useful.

The MD simulations did not show that $\mathrm{CO}_{2}$ has a strongly preferred adsorption site, i.e. atom type, on the PDMS surface. This is expected for gas-polymer combinations with weak interactions and supports our assumption that every surface atom is an available binding site in the reaction-diffusion simulations. If strong hydrogen bonding were possible, the surface area available for adsorption would be reduced. ${ }^{39-40}$ On the other hand, if roughness were significant the surface sites available would be greater than assumed. The MD simulations show that the ratio of the instantaneous surface area to the nominal surface area gives a roughness of 1.1, 

(a)

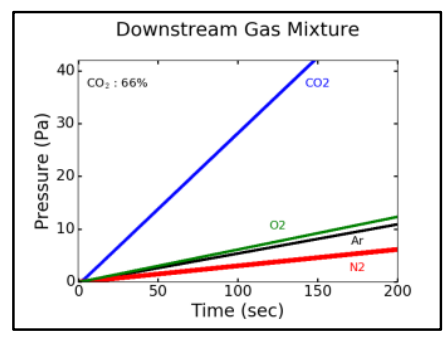

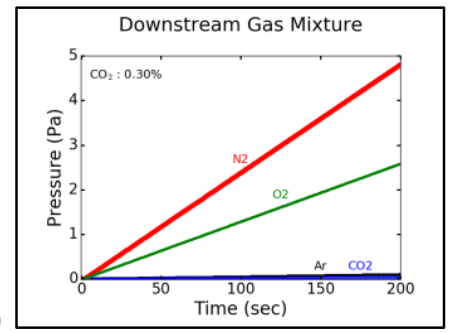

(c)

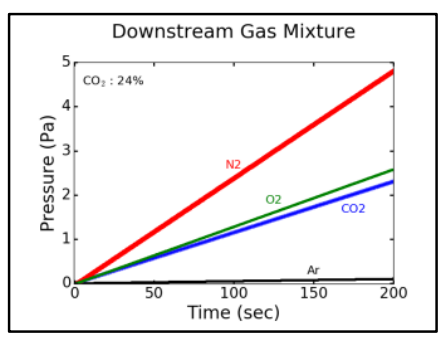

649 Figure 9. Permeation for several mixtures of gases. The permeation of a mixture of gases 650 depends on the upstream composition and the permeability within the polymer, here PDMS. For 651 a mixture in which all components are present in the concentrations (a), the permeation is 652 dominated by the most permeable gas. However, if the most permeable gas is present in only 653 small amounts, then species with lower permeability but higher concentration can dominate. For 654 a mixture with the same composition as air (b), $\mathrm{N}_{2}$ is able to permeate most effectively because it 655 is present in the highest concentration. Even if the permeability of $\mathrm{CO}_{2}$ is increased by 100 times 656 (and holding the other permeabilities constant) (c), the downstream mixture is still only 24\% $657 \mathrm{CO}_{2}$. 


\section{Simulations of solar $\mathrm{CO}_{2}$ reduction product crossover between electrode chambers during a diurnal cycle}

Solar fuels generators utilize membranes to separate the cathode and anode regions, and enable ion transfer between catholyte and anolyte during operation. For an example of the device architecture, see Reference ${ }^{45}$. Reduced products are formed on the cathode side, and oxygen is formed on the anode side. The basic architecture of the commercial membranes used for solar hydrogen generators is a phase-separated polyelectrolyte with glassy regions that are impermeable to $\mathrm{H}_{2}$ and $\mathrm{O}_{2}$, typically dissolved in electrolyte or present as gas bubbles, and hydrated channels with good ionic conductivity. FH-REF A recent modeling study has shown that the $\mathrm{H}_{2}$ and $\mathrm{O}_{2}$ product crossover-blocking property of a membrane for a photoelectrochemical hydrogen generation system is crucial for efficient operation, and can be achieved by reducing conductivity since the crossover path is typically through the hydrated ionic conduction channels. FH-REF

Solar photoelectrochemical devices that convert $\mathrm{CO}_{2}$ into reduced hydrocarbon and oxygenate products present a much more complicated situation. They usually generate mixtures on the cathode side, where each product may influence the others' transport processes through the membrane, and $\mathrm{O}_{2}$ on the anode side, which can diffuse in the opposing direction. FH-REF In a solar $\mathrm{CO}_{2}$ reduction device, the rate of product formation and the chemical composition of the products are voltage-dependent. The cell voltage is determined by the amount of sunlight incident on the light absorber in the device, varying with time of day, season, and weather. FHREFS It cannot be assumed that steady-state membrane operation is ever achieved; however multiphysics models generally do so because of the lack of information about their non-steadystate transport. FH-REFS The glassy matrix blocks significant permeant crossover, however exposure of glassy polymers to $\mathrm{CO}_{2}$ can cause them to plasticize, resulting in development of a rubbery character over time with increased permeability, and product crossover between the electrodes chambers. FH-REFS This will potentially reduce efficiency and selectivity of these systems. Modeling studies examining optimum membrane characteristics for $\mathrm{CO}_{2}$ reduction systems have not been reported to our knowledge.

The model developed in the present work for PDMS permeated by $\mathrm{CH}_{4}$, a typical $\mathrm{CO}_{2}$ reduction product, allows aspects of varying production rate and cross-over to be examined. Assumption of PDMS-like properties is a worst-case scenario, representing a membrane that has been extensively plasticized. The primary modification of the kinetic processes in the model is to change it from a gas-membrane system to an aqueous electrolyte-membrane system. Under such conditions, the interfacial interactions change, but transport within the membrane is unaltered. The surface adsorption rate constant $k_{a d s}$ (see Reaction I) is calculated from the Smoluchowski equation for diffusion-controlled reactions, rather than as a scattering process as in the gaspolymer case. Using the diffusion coefficient of methane in water of $2.35 \times 10^{-9} \mathrm{~m}^{2} / \mathrm{s}$ (at 308 $\mathrm{K})^{46}$ and an interaction distance of $4 \AA$, which is twice the van der Waals radius, then $k_{\text {ads }}=7 \times$ $10^{9} \mathrm{M}^{-1} \mathrm{~s}^{-1}$. The diffusion coefficient of $\mathrm{CH}_{4}$ molecules between electrolyte and interface compartments is set to $1 \mathrm{~m}^{2} / \mathrm{s}$ to represent a rapidly stirred electrolyte. Once the permeant has traversed the membrane, it is removed from the downstream electrolyte. 
At a typical current density for a solar photoelectrochemical device of $0.010 \mathrm{Amp} / \mathrm{cm}^{2}$, if only methane is produced, its production rate is $13 \times 10^{-9} \mathrm{~mol} / \mathrm{cm}^{2} / \mathrm{s}$, where the geometric area of the cathode is used..$^{47}$ For the simulation, an area of $1 \mathrm{~cm}^{2}$ is used for both cathode and membrane; an electrolyte region of $0.1 \mathrm{~cm}$ thickness is used. For this surface area and electrolyte volume, the $\mathrm{CH}_{4}$ production rate per area converts to a volumetric production rate of $1.30 \times 10^{-4}$ $\mathrm{M} / \mathrm{s}$. In the simulation, this is the maximum rate of $\mathrm{CH}_{4}$ production, which is proportional to the amount of sunlight, described as a simple triangle wave lasting a total of 8 hours and shown in Figure 10a. An extended period of darkness follows, in order to measure the amount of time needed for all the $\mathrm{CH}_{4}$ produced to cross over the membrane. The methane production reaction is implemented as the pseudo- $0^{\text {th }}$ order reaction

$$
\text { cathode } \stackrel{k_{\text {prod }}}{\longrightarrow} \text { cathode }+\mathrm{CH}_{4}
$$

where the rate of reaction is the rate coefficient $k_{\text {prod }}$ multiplied by the amount of sunlight. This method is used because the exact concentrations of species and rate coefficients at the cathode surface are unknown. Once produced, methane may become hydrated,

$$
\mathrm{CH}_{4}+\mathrm{aq}-\text { site } \stackrel{k_{h y d}}{\longrightarrow} \mathrm{CH}_{4(\mathrm{aq})}
$$

or bubble out of solution as a gas

$$
\mathrm{CH}_{4} \stackrel{k_{\text {bub }}}{\longrightarrow} \mathrm{CH}_{4(\mathrm{~g})}
$$

where the rate constants for hydration, $k_{\text {hyd }}=10^{50} \mathrm{M} / \mathrm{s}$, and bubbling out, $k_{b u b}=10^{25} \mathrm{M} / \mathrm{s}$, are for nearly instantaneous processes. Reaction VIII is necessary to restrain the $\mathrm{CH}_{4}$ concentration to its solubility limit in water of $0.001 \mathrm{M}$ (for $308 \mathrm{~K}$ ). ${ }^{48}$ It is assumed that the electrolyte is rapidly stirred such that boundary layer and mass transport limitations are negligible.

The transport properties of $\mathrm{CH}_{4}$ in PDMS and two hypothetical polymers (Hyp1 and Hyp2) are listed in Table 5. Both hypothetical polymers have a permeability three orders of magnitude lower than PDMS. For Hyp1, all reduction in permeability comes from a reduction in the diffusivity; for Hyp2, all reduction in permeability comes from a reduction in the solubility. As a point of reference, the values of $\mathrm{D}$ and $\mathrm{S}$ for Hyp1 are similar to those for polystyrene. ${ }^{49}$

Table 5. Properties of the membranes used in the diurnal cycle simulations. The units for solubility are moles/Liter inside of the polymer per moles/Liter in the external electrolyte. The maximum concentration of methane in the electrolyte is 0.001 moles/Liter. Values for PDMS are from Reference ${ }^{33}$. The values for PS are taken from Reference ${ }^{49}$

\begin{tabular}{|l|l|l|l|}
\hline Polymer & $\mathrm{S}$ & $\mathrm{D}$ & $\mathrm{P}$ \\
\hline & $\mathrm{M} / \mathrm{M}$ & $\mathrm{m}^{2} / \mathrm{s}$ & $\mathrm{m}^{2} / \mathrm{s}$ \\
\hline PDMS & 0.467 & $2.20 \times 10^{-9}$ & $1.03 \times 10^{-9}$ \\
\hline PS (Hyp1) & $0.516(0.467)$ & $6.83 \times 10^{-12}\left(2.20 \times 10^{-12}\right)$ & $3.52 \times 10^{-12}\left(1.03 \times 10^{-12}\right)$ \\
\hline Hyp2 & $4.67 \times 10^{-4}$ & $2.20 \times 10^{-9}$ & $1.03 \times 10^{-12}$ \\
\hline
\end{tabular}


(a)

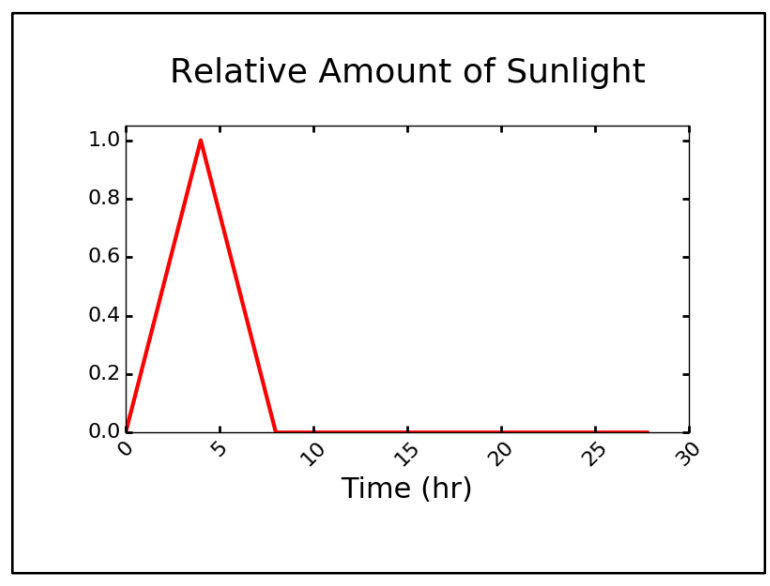

(b)

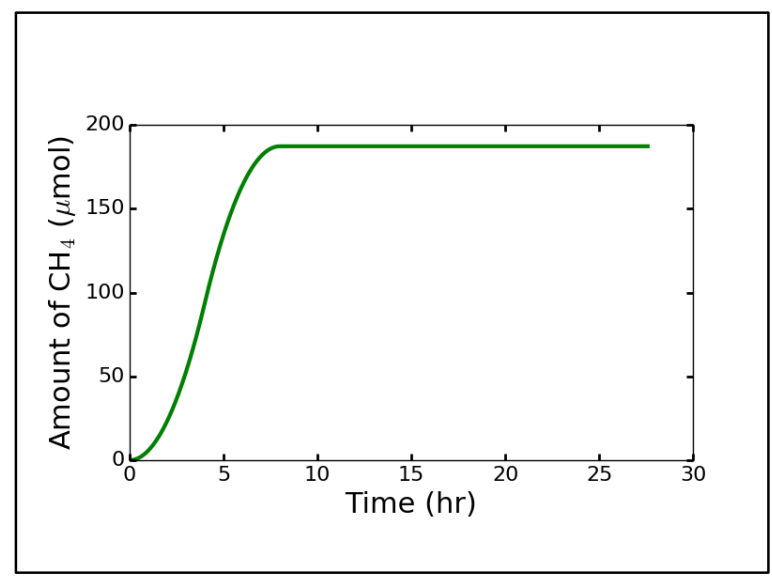

732 Figure 10. The production of $\mathrm{CH}_{4}$. (a) The normalized level of sunlight, which is a multiplier for the rate constant for $\mathrm{CH}_{4}$ production. (b) The total amount of $\mathrm{CH}_{4}$ produced.

The amount of $\mathrm{CH}_{4}$ produced over time is shown in Figure 10b and is the same for all three simulations. The rate of $\mathrm{CH}_{4}$ production increases over the first four hours, reaches its maximum at the peak of available sunlight, then declines over the next four hours, resulting in a total production of $1.87 \times 10^{-4}$ moles of $\mathrm{CH}_{4}$. Because the solubility limit for gases in water is low, a constant aqueous concentration of $\mathrm{CH}_{4}$ is rapidly established, as shown for PDMS, Hyp1, and Hyp2 in Figure 11a, 11d, and 11g, respectively. Additional $\mathrm{CH}_{4}$ produced bubbles out as a gaseous product, as shown for PDMS, Hyp1, and Hyp2 in Figure 11b, 11e, and 11h, respectively. For a theoretical completely blocking membrane, the maximum aqueous concentration is reached in 7.8 minutes. Hyp1 and Hyp2 come close to this, with aqueous phase saturation occurring within 10 minutes. The concentration profile of $\mathrm{CH}_{4}$ within the hypothetical membranes also reaches steady state within 10 minutes. In PDMS, these processes take 20 minutes. The delay in reaching steady state, compared to the hypothetical membranes, is due to the high permeability of PDMS. As $\mathrm{CH}_{4}$ is entering the aqueous phase near the cathode, it is also being removed by transport across the membrane, thus delaying the build-up to its maximum concentration. However, this build-up is only a small fraction of the device's operating time. This is an interesting situation in that, though the solar-fuels device is operating under continually changing conditions, the membrane is essentially experiencing steady-state conditions with respect to this permeant, during daylight hours. 
Once $\mathrm{CH}_{4}$ production ceases, it takes only 3 minutes for the remaining $\mathrm{CH}_{4}$ to traverse

(a)

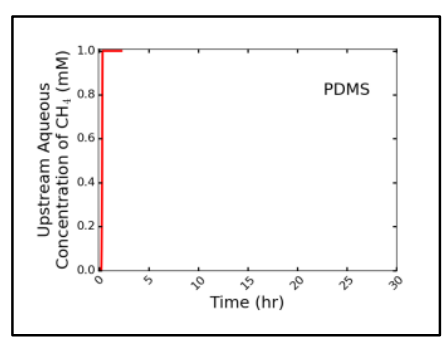

(b)

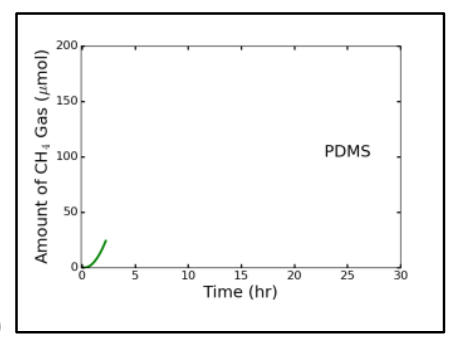

(c)

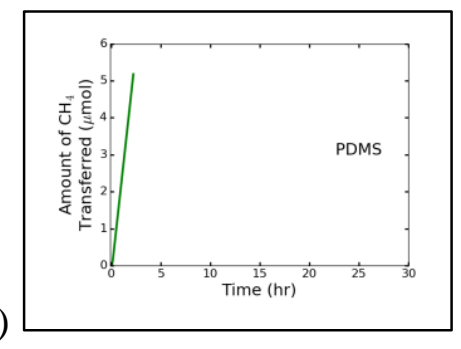

(e)

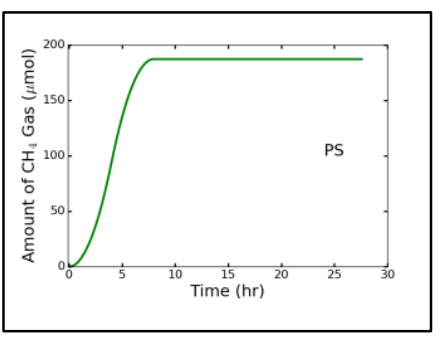

(f)

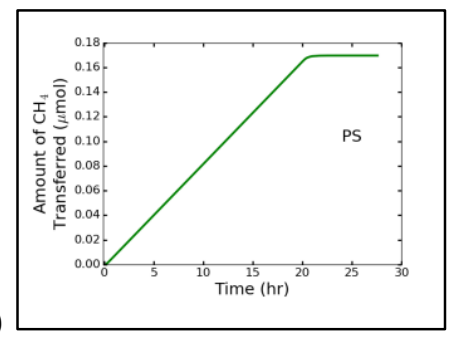

(i) (g)

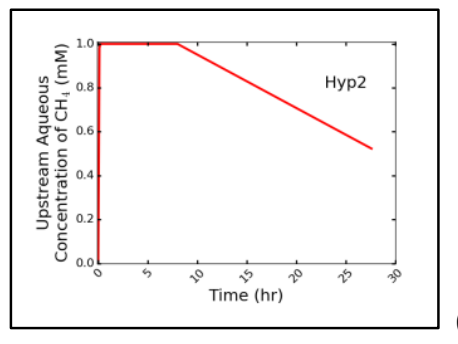

(h)

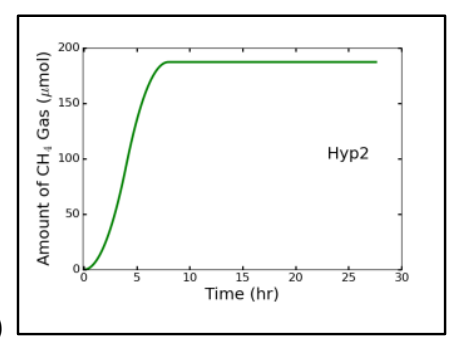

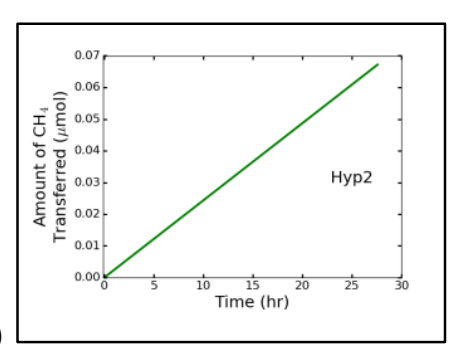


Figure 11. Results of the diurnal simulations. (a) - (c) PDMS, (d) - (f) PS, (g) - (i) Hyp2. (a), (d), (g) Upstream concentration of aqueous $\mathrm{CH}_{4}$. (b), (e), (h) Amount of gaseous $\mathrm{CH}_{4}$ produced. (c), (f), (i) Amount of $\mathrm{CH}_{4}$ cross-over.

\section{Conclusions}

In this work we describe a multiscale modeling study of permeation of polymer membranes by simple solutes, with the goal of developing a full description of steady-state and non-steadystate processes. We have chosen to focus on permeation of rubbery polymers by inert gases as the simplest case since the polymer transport properties are expected to be constant over time. We have confirmed that the permeation process is insensitive to permeant adsorption and absorption kinetics in these systems, consistent with the assumption made in the solutiondiffusion model for membrane permeation. Two quantitatively validated models are reported built on a common reaction-diffusion framework: quenching of PtOEP phosphorescent dye in C4PTP, an oxygen sensor system, and permeation of $\mathrm{O}_{2}, \mathrm{~N}_{2}, \mathrm{CH}_{4}$ and $\mathrm{CO}_{2}$ through a PDMS separator. The framework is extended to simulate two additional hypothetical systems, separation of $\mathrm{CO}_{2}$ from air using a PDMS-like membrane, and crossover of the $\mathrm{CO}_{2}$ reduction product, $\mathrm{CH}_{4}$, in a solar fuels system whose membrane separator is rubbery or glassy.

Because the goal is development of a quantitatively predictive model with no adjustable parameters, the model construction process is dependent on time-dependent permeation data as well as experimental and theoretical information on specific materials properties and processes. Such data are not commonly available in the literature so experimental and MD computational studies have been performed and are reported in this paper. The results show that a simple reaction-diffusion framework simulated using stochastic methods is adequate to capture the details of interfacial processes as well as bulk processes, covering a broad range of length and timescales. Measured quantities such as solubilities and diffusion coefficients contain enough information about solute-polymer interactions to predict experimental observables such as pressure increases and phosphorescence quenching without explicit inclusion of free volume dynamics and tortuosity, although these elements can be built into the model with straightforward modifications.

Several new insights to polymer-permeant systems have been obtained through the simulations.

(i) Time-dependent phosphorescence quenching is related in a complicated way to the amount of $\mathrm{O}_{2}$ sorbed into a polymer due to the rate mismatch between dye- $\mathrm{O}_{2}$ interactions and $\mathrm{O}_{2}$ transport. This suggests that such sensors are best used in conjunction with models such as that presented here to ensure complete data interpretation.

(ii) The pre-steady state portion of a gas permeation curve contains a great deal of information about the nature of the gas-polymer interaction. In particular, we find it necessary to include a dynamically increasing maximum concentration within the polymer as the upstream permeant pressure increases, in accordance with Henry's law solubility, to successfully reproduce the experimental data. This raises interesting questions about what is occurring dynamically inside the polymer, especially how interfacial processes affect the polymer bulk, and how fast this response is. 
(iii) The behavior of low-permeability membranes during both steady state and non-steady state contribute to their blocking effects. It is likely that successful membrane and separation systems design for complex situations such as solar fuels generators and $\mathrm{CO}_{2}$ capture will require integration of models such as those outlined here with experiment.

826 Table 6. All the symbols, their meanings and units, used in this study. The mark - indicates that 827 the quantity is unitless.

\begin{tabular}{|l|l|l|}
\hline Symbol & Meaning & $\mathrm{Unit}$ \\
\hline$\rho$ & mass density & $\mathrm{kg} / \mathrm{m}^{3}$ \\
\hline $\mathrm{P}_{\mathrm{m}}$ & permeability of the membrane & $(\mathrm{mol} \mathrm{m}) /\left(\mathrm{m}^{2} \mathrm{~s} \mathrm{~Pa}\right)$ for gas phase \\
\hline & & $\mathrm{m}^{2} / \mathrm{s}$ for liquid phase \\
\hline $\mathrm{J}$ & flux through membrane & $\mathrm{mol} /\left(\mathrm{m}^{2} \mathrm{~s}\right)$ \\
\hline$l$ & thickness of the membrane & $\mathrm{m}$ \\
\hline $\mathrm{A}$ & area & $\mathrm{m}^{2}$ \\
\hline $\mathrm{R}$ & universal gas constant & $\mathrm{J} /(\mathrm{mol} \mathrm{K})$ \\
\hline $\mathrm{T}$ & temperature & $\mathrm{K}$ \\
\hline $\mathrm{p}$ & pressure & $\mathrm{Pa}$ \\
\hline $\mathrm{t}$ & time & $\mathrm{s}$ \\
\hline $\mathrm{V}$ & volume & $\mathrm{m}^{3}$ \\
\hline $\mathrm{D}$ & diffusivity & $\mathrm{m}^{2} / \mathrm{s}$ \\
\hline $\mathrm{S}$ & solubility & $\mathrm{mol} /(\mathrm{L} \mathrm{Pa})$ for gas phase \\
\hline & & $(\mathrm{mol} / \mathrm{L}) /(\mathrm{mol} / \mathrm{L})$ for liquid phase \\
\hline $\mathrm{k}$ & rate constant & $\mathrm{M}^{\text {-a }} \mathrm{s}^{-1} *$ \\
\hline$\mu$ & sticking probability & -- \\
\hline $\mathrm{Z}$ & collision frequency & $\mathrm{s}^{-1}$ \\
\hline $\mathrm{k}_{\mathrm{B}}$ & Boltzmann constant & $\mathrm{J} / \mathrm{K}$ \\
\hline$\pi$ & pi & -- \\
\hline $\mathrm{m}_{\mathrm{W}}$ & molecular mass & $\mathrm{kg} / \mathrm{mol}$ \\
\hline $\mathrm{N}$ & number of molecules & -- \\
\hline $\mathrm{I}$ & intensity of light emission & $--\mathrm{m}^{2}$ \\
\hline$\sigma_{\mathrm{a}}$ & absorption cross section & $\mathrm{J} \mathrm{m}^{2}$ \\
\hline$\omega_{\mathrm{L}}$ & energy density & \\
\hline & & \\
\hline
\end{tabular}




\begin{tabular}{|l|l|l|}
\hline $\mathrm{h}$ & Planck constant & $\mathrm{J} \mathrm{s}$ \\
\hline$v_{\mathrm{L}}$ & frequency of exciting light & $\mathrm{m}$ \\
\hline$\phi_{\mathrm{P}}$ & phosphorescent quantum yield & -- \\
\hline$\tau$ & lifetime & $\mathrm{s}$ \\
\hline$[\mathrm{X}]$ & concentration of $\mathrm{X}$ & $\mathrm{mol} / \mathrm{L}$ \\
\hline $\mathrm{B}+1$ & ratio of intensities & -- \\
\hline$\xi$ & relative concentration & -- \\
\hline $\mathrm{Q}$ & absolute concentration & $\mathrm{mol} / \mathrm{L}$ \\
\hline $\mathrm{X}$ & position & $\mathrm{m}$ \\
\hline $\mathrm{R}_{\mathrm{AB}}$ & encounter distance & $\mathrm{m}$ \\
\hline $\mathrm{N}_{\mathrm{Av}}$ & Avogadro's number & $\mathrm{molecules} / \mathrm{mole}$ \\
\hline
\end{tabular}

* with a being the reaction order

$829 * *$ All reported intensities are normalized and so unitless.

\section{Supplementary Information}

832 Supplementary data related to this article can be found at http:// link

833 Data tables for all figures and additional experimental data can be found in the spreadsheet at 834 link

\section{Acknowledgments}

837 This material is based upon work performed by the Joint Center for Artificial Photosynthesis, a 838 DOE Energy Innovation Hub, as follows: The reaction-diffusion simulations and experimental measurements were performed by M. S., F. H., M. T. and A. W. supported through the Office of

840 Science of the U.S. Department of Energy under Award Number DE-SC0004993. The MD

841 simulation work by D. B., B. M. and W. A. G. was supported by institution under Award

842 Number XXXX. M. T. thanks the National Science Foundation NEED DETAILS. The authors

843 are grateful to Dr. Daniel Miller (JCAP, LBNL) for many helpful discussions on membrane

844 polymer science, Mr. Ezra L. Clark for data on photoelectrochemical production of methane, and 845 to Dr. William D. Hinsberg (Columbia Hill Technical Consulting) for discussions on the use of 846 Kinetiscope in this work. 
850 1. Mulder, M., Basic Principles of Membrane Technology. Kluwer Academic Publishers: 8511996.

852 2. Wijmans, J. G.; Baker, R. W., The Solution-Diffusion Model - a Review. J Membrane 853 Sci 1995, 107 (1-2), 1-21.

854 3. Crank, J.; Park, G. S., Diffusion in Polymers. Academic Press Inc., Ltd.: London and 855 New York, 1968.

856 4. Shin, D. W.; Guiver, M. D.; Lee, Y. M., Hydrocarbon-Based Polymer Electrolyte 857 Membranes: Importance of Morphology on Ion Transport and Membrane Stability. Chem Rev $858 \quad 2017,117(6), 4759-4805$.

859 5. Kusoglu, A.; Weber, A. Z., Study of PFSA ionomers using X-ray scattering techniques. 860 Abstr Pap Am Chem S 2014, 248.

861 6. Jayarajah, C. N. Luminescence Quenching Studies of Oxygen Dinusion in Highly 862 Permeable Media. 1998.

863 7. Jayarajah, C. N.; Yekta, A.; Manners, I.; Winnik, M. A., Oxygen diffusion and 864 permeability in alkylaminothionylphosphazene films intended for phosphorescence barometry 865 applications. Macromolecules 2000, 33 (15), 5693-5701.

866 8. Wang, X.-d.; Wolfbeis, O. S., Optical methods for sensing and imaging oxygen: 867 materials, spectroscopies and applications. Chemical Society Reviews 2014, 43, 3666-761.

$8689 . \quad$ Bunker, D.; Garrett, B.; Kleindienst, T.; III, G. L., Discrete simulation methods in 869 combustion kinetics. Combustion and Flame 1974, 23, 373-379.

870 10. Gillespie, D. T., General Method for Numerically Simulating Stochastic Time Evolution 871 of Coupled Chemical-Reactions. J Comput Phys 1976, 22 (4), 403-434.

872 11. Hinsberg, W. D.; Houle, F. A., Kinetiscope. www.hinsberg.net/kinetiscope. , 2015.

873 12. Houle, F. A.; Hinsberg, W. D.; Morrison, M.; Sanchez, M. I.; Wallraff, G.; Larson, C.; 874 Hoffnagle, J., Determination of coupled acid catalysis-diffusion processes in a positive-tone 875 chemically amplified photoresist. Journal of Vacuum Science \& Technology B: Microelectronics 876 and Nanometer Structures 2000, 18, 1874.

877 13. Houle, F. A.; Hinsberg, W. D.; Sanchez, M. I., Kinetic Model for Positive Tone Resist 878 Dissolution and Roughening. Macromolecules 2002, 35, 8591-8600.

879 14. Wiegel, A. A.; Wilson, K. R.; Hinsberg, W. D.; Houle, F. A., Stochastic methods for 880 aerosol chemistry: a compact molecular description of functionalization and fragmentation in the 881 heterogeneous oxidation of squalane aerosol by $\mathrm{OH}$ radicals. Phys. Chem. Chem. Phys. 2015, 17, 882 4398-4411.

883 15. Wiegel, A. A.; Liu, M.; Hinsberg, W. D.; Wilson, K. R.; Houle, F. A., Diffusive 884 Confinement of Free Radical Intermediates in the OH Radical Oxidation of Semisolid Aerosol. 885 Physical Chemistry Chemical Physics 2017, 19, 6814-6830. 
16. McQuarrie, D. A., Statistical Mechanics. University Science Books: Mill Valley, CA, 2000; p 641-641.

17. Martyna, G. J.; Tobias, D. J.; Klein, M. L., Constant pressure molecular dynamics algorithms. Journal of Chemical Physics 1994, 101 (5), 4177-4177.

18. Shin, H.; Pascal, T. A.; Goddard, W. A.; Kim, H., Scaled effective solvent method for predicting the equilibrium ensemble of structures with analysis of thermodynamic properties of amorphous polyethylene glycol-water mixtures. Journal of Physical Chemistry B 2013, 117 (3), 916-927.

\section{Polymer Data Handbook. Oxford University Press: 1999; p 1012-1012.}

20. Willard, A. P.; Chandler, D., Instantaneous liquid interfaces. Journal of Physical Chemistry B 2010, 114 (5), 1954-1958.

21. Julin, J.; Shiraiwa, M.; Miles, R. E. H.; Reid, J. P.; Pöschl, U.; Riipinen, I., Mass accommodation of water: Bridging the gap between molecular dynamics simulations and kinetic condensation models. Journal of Physical Chemistry A 2013, 117 (2), 410-420.

22. Julin, J.; Winkler, P. M.; Donahue, N. M.; Wagner, P. E.; Riipinen, I., Near-Unity Mass Accommodation Coefficient of Organic Molecules of Varying Structure. Environmental Science \& Technology 2014, 48, 12083-12089.

23. Springer Handbook of Materials Measurement Methods. Springer: Berlin, 2006; p 1207.

24. Baratron General Purpose Capacitance Manometers: 750C11T. .

25. Engel, T.; Reid, P., Thermodynamics, Statistical Thermodynamics, \& Kinetics. 2nd ed.; Prentice Hall: 2010.

26. Somorjai, G. A.; Li, Y., Introduction to Surface Chemistry and Catalysis. Wiley: 2010; p 800-800.

27. Atkins, P.; de Paula, J., Physical Chemistry. 8th ed.; W. H. Freeman and Co.: New York, USA, 2006.

28. Houston, P., Chemical kinetics and reaction dynamics. DOVER PUBLICATIONS, INC.: Mineola, New York, 2001; p 48-49.

29. Bansal, A. K.; Holzer, W.; Penzkofer, A.; Tsuboi, T., Absorption and emission spectroscopic characterization of platinum-octaethyl-porphyrin (PtOEP). Chemical Physics 2006, 330 (1-2), 118-129.

30. Dienel, T.; Proehl, H.; Fritz, T.; Leo, K., Novel near-infrared photoluminescence from platinum(II)-porphyrin (PtOEP) aggregates. Journal of Luminescence 2004, 110, 253-257.

31. Hodge, K.; Prodpran, T.; Shenogina, N. B.; Nazarenko, S., Diffusion of oxygen and carbon dioxide in thermally crystallized syndiotactic polystyrene. Journal of Polymer Science Part B: Polymer Physics 2001, 39, 2519-2538.

32. Yekta, A.; Masoumi, Z.; Winnik, M. A., Luminescence measurements of oxygen permeation and oxygen diffusion in thin polymer films. Canadian Journal of Chemistry 1995, 73 (11), 2021-2029. 
924

925

33. Merkel, T. C.; Bondar, V. I.; Nagai, K.; Freeman, B. D.; Pinnau, I., Gas sorption, diffusion, and permeation in poly(dimethylsiloxane). Journal of Polymer Science, Part B: Polymer Physics 2000, 38 (3), 415-434.

34. Brandão, L.; Madeira, L. M.; Mendes, A. M., Mass transport on composite dense PDMS membranes with palladium nanoclusters. Journal of Membrane Science 2007, 288 (1-2), 112122.

35. Berean, K.; Ou, J. Z.; Nour, M.; Latham, K.; McSweeney, C.; Paull, D.; Halim, A.; Kentish, S.; Doherty, C. M.; Hill, A. J.; Kalantar-zadeh, K., The effect of crosslinking temperature on the permeability of PDMS membranes: Evidence of extraordinary $\mathrm{CO} 2$ and $\mathrm{CH} 4$ gas permeation. Separation and Purification Technology 2014, 122, 96-104.

36. Yampolskii, Y. P.; Pinnau, I.; Freeman, B. D., Materials Science of Membranes for Gas and Vapor Separation. John Wiley \& Sons Ltd: West Sussex, England, 2006; p 445-445.

37. Lancaster, D. K.; Johnson, A. M.; Burden, D. K.; Wiens, J. P.; Nathanson, G. M., Inert gas scattering from liquid hydrocarbon microjets. Journal of Physical Chemistry Letters 2013, 4 (18), 3045-3049.

38. Alexander, W. a.; Zhang, J.; Murray, V. J.; Nathanson, G. M.; Minton, T. K., Kinematics and dynamics of atomic-beam scattering on liquid and self-assembled monolayer surfaces. Faraday Discussions 2012, 157, 355-374.

39. Donaldson, D. J., Adsorption of Atmospheric Gases at the Air-Water Interface. I. NH 3. Journal of Physical Chemistry A 1999, 103 (1), 62-70.

40. Ogasawara, H.; Horimoto, N.; Kawai, M., Ammonia adsorption by hydrogen bond on ice and its solvation. Journal of Chemical Physics 2000, 112 (19), 8229-8232.

41. House, K. Z.; Baclig, A. C.; Ranjan, M.; van Nierop, E. A.; Wilcox, J.; Herzog, H. J., Economic and energetic analysis of capturing $\mathrm{CO} 2$ from ambient air. Proceedings of the National Academy of Sciences 2011, 108 (51), 20428-20433.

42. Jones, C. W., $\mathrm{CO}(2)$ capture from dilute gases as a component of modern global carbon management. Annual review of chemical and biomolecular engineering 2011, 2, 31-52.

43. Dhingra, S. S.; Marand, E., Mixed gas transport study through polymeric membranes. Journal of Membrane Science 1998, 141, 45-63.

44. Wu, F.; Li, L.; Xu, Z.; Tan, S.; Zhang, Z., Transport study of pure and mixed gases through PDMS membrane. Chemical Engineering Journal 2006, 117, 51-59.

45. Singh, M. R.; Clark, E. L.; Bell, A. T., Effects of electrolyte, catalyst, and membrane composition and operating conditions on the performance of solar-driven electrochemical reduction of carbon dioxide. Physical Chemistry Chemical Physics 2015, 17 (29), 18924-18936.

46. Jähne, B.; Heinz, G.; Dietrich, W., Measurement of the diffusion coefficients of sparingly soluble gases in water. Journal of Geophysical Research: Oceans 1987, 92 (C10), 10767-10776.

47. Clark, E. L., personal communication.

48. Pergamon Press: Oxford, England, 1987; Vol. 27/28, Methane.

49. Rein, D. H.; Baddour, R. F.; Cohen, R. E., Gas solubility and diffusion in a polystyrenepolybutadiene block copolymer. Journal of Applied Polymer Science 1992, 45 (7), 1223-1227. 
964 